Published in final edited form as:

J Fluency Disord. 2014 September ; 0: 12-31. doi:10.1016/j.jfludis.2014.06.002.

\title{
Autonomic nervous system activity of preschool-age children who stutter
}

\author{
Robin M. Jones, Anthony P. Buhr, Edward G. Conture, Victoria Tumanova, Tedra A. \\ Walden, and Stephen W. Porges \\ Robin M. Jones, Department of Hearing and Speech Sciences, Vanderbilt University; Anthony P. \\ Buhr, University of Alabama; Edward G. Conture, Department of Hearing and Speech Sciences, \\ Vanderbilt University; Victoria Tumanova, Department of Communication Sciences and \\ Disorders, Syracuse University; Tedra A. Walden, Department of Psychology and Human \\ Development, Vanderbilt University; Stephen W. Porges, Department of Psychiatry, University of \\ North Carolina at Chapel Hill
}

\begin{abstract}
Purpose-The purpose of this study was to investigate potential differences in autonomic nervous system (ANS) activity to emotional stimuli between preschool-age children who do (CWS) and do not stutter (CWNS).

Methods-Participants were 20 preschool-age CWS (15 male) and 21 preschool-age CWNS (11 male). Participants were exposed to two emotion-inducing video clips (negative and positive) with neutral clips used to establish pre-and post-arousal baselines, and followed by age-appropriate
\end{abstract}

\footnotetext{
(C) 2014 Elsevier Inc. All rights reserved

Correspondence concerning this article should be addressed to Robin M. Jones, Department of Hearing and Speech Sciences, Vanderbilt University, 10221 MCE South Tower, $121521^{\text {st }}$ Avenue South, Nashville, TN 37232. Phone: 615-875-1184, robin.m.jones@vanderbilt.edu..

Robin M. Jones, Ph.D., Assistant Professor, Hearing and Speech Sciences, Vanderbilt University, Nashville, TN 37232 . Jones's primary research interest relate to childhood stuttering, with a focus on emotional and linguistic contributions to stuttering as well as empirical assessment and treatment of stuttering.

Anthony Buhr is an assistant professor at the University of Alabama, Tuscaloosa. He researches the organization of behavior in social contexts.

Edward G. Conture, PhD., Professor Emeritus, Hearing and Speech Sciences, Vanderbilt University, Nashville, TN 37232. Conture has authored over 130 peer-reviewed papers, several books and given over 300 professional/scholar presentations to (inter)national conferences. Conture's main research interests relate to childhood stuttering, particularly emotional/psycholinguistic contributions. Victoria Tumanova earned her Ph.D. in Speech and Hearing Science from the University of Iowa in 2010. She is now an Assistant Professor in the Department of Communication Sciences and Disorders at Syracuse University. Her research interests include the development of early childhood stuttering and its physiological correlates.

Tedra A. Walden is Professor of Psychology and Hearing and Speech Sciences at Vanderbilt University and head of the Developmental Sciences Program. Her research focuses on social and emotional development of young children, especially children with developmental disabilities.

Stephen Porges is Research Professor of Psychology at Northeastern University, Adjunct Professor of Psychiatry at the University of North Carolina, Professor Emeritus of Psychiatry at the University of Illinois at Chicago, and Professor Emeritus at the University of Maryland. He is a former president of the Society for Psychophysiological Research.

Publisher's Disclaimer: This is a PDF file of an unedited manuscript that has been accepted for publication. As a service to our customers we are providing this early version of the manuscript. The manuscript will undergo copyediting, typesetting, and review of the resulting proof before it is published in its final citable form. Please note that during the production process errors may be discovered which could affect the content, and all legal disclaimers that apply to the journal pertain.

Educational objectives: The reader will be able to: (1) summarize current empirical evidence on the role of emotion in childhood stuttering; (2) describe physiological indexes of sympathetic and parasympathetic activity; (3) summarize how preschool-age children who stutter differ from preschool-age children who do not stutter in autonomic activity; (4) discuss possible implications of current findings in relation to the development of childhood stuttering.
} 
speaking tasks. Respiratory sinus arrhythmia (RSA) - often used as an index of parasympathetic activity - and skin conductance level (SCL) - often used as an index of sympathetic activity were measured while participants listened to/watched the audio-video clip presentation and performed a speaking task.

Results-CWS, compared to CWNS, displayed lower amplitude RSA at baseline and higher SCL during a speaking task following the positive, compared to the negative, condition. During speaking, only CWS had a significant positive relation between RSA and SCL.

Conclusion-Present findings suggest that preschool-age CWS, when compared to their normally fluent peers, have a physiological state that is characterized by a greater vulnerability to emotional reactivity (i.e., lower RSA indexing less parasympathetic tone) and a greater mobilization of resources in support of emotional reactivity (i.e., higher SCL indexing more sympathetic activity) during positive conditions. Thus, while reducing stuttering to a pure physiological process is unwarranted, the present findings suggest that parasympathetic and sympathetic nervous system activity is involved.

\section{Keywords}

stuttering; preschool-age; respiratory sinus arrhythmia; skin conductance

\section{Introduction}

The association between emotional processes and developmental stuttering has attracted increasing interest over the past decade (e.g., Anderson, Pellowski, Conture \& Kelly, 2003; Arnold, Conture, Key, \& Walden, 2011; Choi, Conture, Walden, Lambert, \& Tumanova, 2013; Eggers, De Nil, \& Van den Bergh, 2009, 2010, 2012; Felsenfeld, van Beijsterveldt \& Boomsma, 2010; Johnson, Walden, Conture, \& Karrass, 2010; Karrass et al., 2006; Ntourou, Conture, \& Walden, 2013; Schwenk, Walden \& Conture, 2007; Walden et al., 2012). Recent reviews of the literature have concluded that there may be a relation between the two (for review, see Conture, Kelly, \& Walden, 2013; Jones, Choi, Conture, \& Walden, in press; Keflianos, Onslow, Block, Menzies, \& Reilly, 2012). Despite inconsistencies in the literature, there are also consistencies. For example, reviews noted that preschool-age children, who stutter (CWS) compared to children who do not stutter (CWNS), exhibit (1) less adaptability, (2) poorer attention span/persistency and attention regulation, and (3) more negative mood. Although these findings do not indicate whether emotions are causes or the consequences of stuttering, they provide replicated support for an association between emotional state regulation and childhood stuttering.

\subsection{Emotion and Stuttering}

1.1.I. Emotions and childhood stuttering-Studies using caregiver report questionnaires documented that CWS, compared to CWNS, displayed: a) less temperamental adaptability, distractibility, and rhythmicity (Anderson et al., 2003), b) increased reactivity and greater difficulty regulating emotions (Karrass et al., 2006), c) poorer attention regulation skills (Felsenfeld, van Beijsterveldt, \& Boomsma, 2010; Karrass et al., 2006), skill implicated in emotion regulation (Rothbart, Ahadi, \& Evans, 2000), and d) less inhibitory control and attention shifting, as well as greater anger/frustration (Eggers, De 
Nil, \& Van den Bergh, 2010). In contrast, Lewis and Goldberg (1997) found that CWS were less negative in their emotions and more adaptable than CWNS. Despite some incongruence, taken as a whole these findings provide building evidence that CWS versus CWNS display consistent differences in a variety of emotion-related processes.

Using direct observation of behavior or experimental testing, researchers report that preschool-age CWS, compared to CWNS, exhibited: a) difficulty habituating to irrelevant background stimuli (Schwenk, Conture, \& Walden, 2007), b) more negative emotional expressions in a disappointing gift procedure (Johnson, Walden, Conture, \& Karrass, 2010) and during neutral and frustrating conditions (Ntourou et al., 2013), c) less efficiency of the orienting subsystem of the attentional system (Eggers, De Nil \& Van den Bergh, 2012; cf. Johnson, Conture, \& Walden, 2012), and d) poorer inhibitory control during a Go/NoGo task (Eggers, De Nil, Van den Bergh, 2013).

Within-group behavioral observations have indicated that increases in CWSs stuttering are related to: a) decreased duration and frequency of behavioral regulatory strategies (Arnold et al., 2011), b) increased emotional reactivity concurrent with decreased regulation (Walden et al., 2012), c) negative emotion prior to and during utterances (Jones, Conture, \& Walden, in press), particularly following positive conditions (Johnson et al., 2010; Jones et al., in press). In contrast, CWSs stuttering frequency was lower during narratives that followed diverting attention away from preceding non-speech tasks (Ntourou et al., 2013). Further, more behaviorally inhibited CWS (i.e., children exhibiting strong reactions to novelty or change), when compared to less behaviorally inhibited CWS, exhibited more stuttering during a conversation with an unfamiliar experimenter (Choi et al., 2013). These behavioral observations provide insights into CWS' emotional responding to specific challenging situations, as well as concomitant changes in speech fluency.

The above findings have resulted in a partial picture of the association between emotion and childhood stuttering. Including psychophysiological methods to assess the two branches (i.e., parasympathetic and sympathetic) of the autonomic nervous system (ANS) may increase our understanding of this association in preschool-age CWS. Measures of autonomic activity would provide an important feature of emotional reactivity and regulation and possibly identify covert indicators that may precede, follow, or act concurrently with more overt expressions of emotion. Since much of the empirical evidence regarding emotion and childhood stuttering comes from parent-report and behavioral observations, a psychophysiological perspective is largely lacking (cf. Arnold et al., 2011). This dearth in knowledge, in part, motivated the present study.

1.1.2. Autonomic nervous system activity of adults who stutter-Despite the lack of knowledge about ANS activity of preschool-age CWS, psychophysiological concomitants of stuttering in adults have been empirically studied for several decades (e.g., Alm \& Risberg, 2007; Fletcher, 1914; Gray \& Karman, 1967; Reed \& Lingwall, 1980). Methods have varied considerably as technology and theories have advanced, which may, in part, contribute to a lack of consistent evidence that psychophysiological factors are associated with stuttering. For example, although adults who stutter often report anxiety about stuttering (e.g., Craig, 1990; Craig, Hancock, Tran, \& Craig, 2003), this is not always 
associated with increases in autonomic arousal (e.g., Dietrich \& Roaman, 2001). This is particularly evident in recent investigations, which have reported heart rate increases either prior to or during speech for both adults who stutter (AWS) and adults who do not stutter (AWNS), but that such increases are often less marked for AWS (Caruso, Chodzko-Zaijko, Bidinger, \& Sommers, 1994; Peters \& Hulstijn, 1984; Weber \& Smith, 1990). Alm (2004) argued that these findings may represent "anticipatory anxiety resulting in a 'freezing response"' that may be related to parasympathetic inhibition of heart rate. Alm further argued that heart rate is not thought to be a reliable index of sympathetic or parasympathetic activity, but rather represents the cumulative influences of both sympathetic and parasympathetic activity. Therefore, in order to attain a more "pure" index of the activity of the two branches of the ANS, measures that isolate specific branches need to be used. These measures should be conceptualized using a well-established framework to understand separate and concurrent influences on psychological and behavioral processes.

\subsection{Psychophysiological Measures of Emotion}

Before introducing the indices of ANS activity used in the present study, we first briefly discuss the role of physiology in emotion. At present there is no consensus in the literature on a definition of emotion. However, Cabanac (2002) proposed that it be operationalized as "any mental experience with high intensity and high hedonic content (pleasure/ displeasure)." Cabanac further predicted that objective physiological signs of emotion should accompany such mental experiences. Cabanac's view corresponds with Sonnemans and Frijda's (1994) view that physiological changes are an important component to the intensity of emotion. Izard (2010) compiled responses to various questions on emotion from 35 "distinguished" emotion researchers, and while the consensus was that there is no unitary concept, physiological activity was commonly included as a defining feature of emotion. Therefore, while we acknowledge that the physiological activity used to assess emotion in the present study does not solely represent emotion, we assert that it is an important component of emotion that furthers our understanding of emotion and childhood stuttering. The importance of physiological activity to a comprehensive account of emotion is underscored (see below) by the Polyvagal Theory (Porges, 2007; 2011), a model providing a conceptual framework for the present study.

1.2.1. Polyvagal theory-The Polyvagal Theory proposes a hierarchical model comprised of three neural circuits that regulate autonomic activity and support distinct behavioral processes (for detailed overview of the Polyvagal Theory, see Porges, 1995, 2007, 2011). In brief, the Polyvagal Theory describes three phylogenetically ordered neural circuits that regulate autonomic state in vertebrates. In humans these circuits are linked behaviorally to (1) social communication (e.g., facial expression, vocalization, listening), (2) mobilization (e.g., fight-flight behaviors), and (3) immobilization (e.g., feigning death and behavioral shutdown) (see Table 1). The Polyvagal Theory proposes an integrated "social communication system" that involves regulation of the striated muscles of the face and head (i.e., via cranial nerves V, VII, IX, X, XI) and regulation of visceral organs above the diaphragm via a myelinated vagal pathway. This vagal circuit fosters calm states by facilitating parasympathetic nervous system influence on the heart and inhibits sympathetic activation. The "mobilization system"—-related to sympathetic nervous system activation- 
promotes "fight-flight" behaviors. Within the Polyvagal Theory, the mobilization system is also characterized by a synchronous withdrawal of the parasympathetic influence on the heart to facilitate an efficient sympathetic activation. Lastly, the earliest, phylogenetically most primitive system is the "immobilization system" which depends on the unmyelinated vagus with its primary regulation of subdiaphragmatic organs (e.g., gut). This system promotes behavioral shutdown in response to life threat and is associated with massive slowing of heart rate (i.e., clinical bradycardia) that would lead to a rapid drop in blood pressure and fainting (i.e., vasovagal syncope).

Based on this hierarchical model, parasympathetic activity (i.e., myelinated vagus) inhibits sympathetic activity and supports spontaneous social engagement behaviors; sympathetic activity inhibits the unmyelinated vagus, which results in an inhibition of digestive processes during the sympathetic activation associated with mobilized behaviors. Thus, decreases in parasympathetic activity are associated with less inhibition of sympathetic nervous system activity. Such lack of inhibition by the "myelinated" vagus may result in increased sympathetic activity, which supports mobilization. All three of these circuits - myelinated vagus ("social engagement system), sympathetic nervous system ("mobilization system"), and unmyelinated vagus ("immobilization system")—are conceptualized as dynamic, providing adaptive responses for a range of safe to challenging to life threatening environmental events or situations.

Since the Polyvagal Theory was proposed in 1995, there have been several thousand citations in peer-reviewed journals. Although criticisms of the Polyvagal Theory have been infrequent, criticism may be helpful in understanding limitations and in identifying future directions for research. For example, Berntson, Cacioppo, \& Grossman (2007) suggest that because the relative contributions of vagal pathways originating from nucleus ambiguus (myelinated vagus) and dorsal motor nucleus (unmyelinated vagus) cannot be invasively or non-invasively assessed, attributions of differential psychophysiological and behavioral functioning in humans remain speculative. However, inference can still be derived from premature infants in which vagal fibers are myelinating and migrating from dorsal motor nucleus of the vagus to nucleus ambiguus. The anatomical literature documents a developmental shift in the distribution of myelinated and unmyelinated vagal fibers in preterm human infants that parallels the developmental increase in RSA and the vulnerability to clinical bradycardia (see Porges \& Furman, 2011). Furthermore, the neurophysiological literature has documented the existence of vagal inhibitory pathways originating from both the nucleus ambiguus (i.e., myelinated vagal pathways) and the dorsal motor nucleus of the vagus (i.e., unmyelinated vagal pathways) terminating on the heart (e.g., Wang, Holst, \& Powley, 1995).

The polyvagal theory has been successfully applied to many behavioral and psychological processes and disorders, both by Porges (2007) and others (e.g., Beauchaine, Gatzke-Kopp, \& Mead, 2007; Hastings, Kahle, Nuselocvici, in press; Utendale et al., 2014), making it a well-regarded, substantive, and relevant theory to apply to the study of parasympathetic and sympathetic activity in childhood stuttering. 


\subsubsection{Respiratory sinus arrhythmia: an index of emotion regulation-}

Respiratory sinus arrhythmia (RSA), a physiological measure linking the function of the myelinated vagus to the heart, provides a psychophysiological index that is central to the Polyvagal Theory. Respiratory sinus arrhythmia can be operationally defined as the amplitude of periodic fluctuations in heart rate occurring at approximately the frequency of spontaneous respiration. RSA is a well-documented measure of the influence of myelinated vagus on the heart (Porges, 1995; Porges, 2007; Lewis et al., 2012). Further, the measure of RSA is a frequently used physiological index that has been reliably linked behavioral and emotional regulation in children (Beauchanie, Gatzke-Kopp, \& Mead, 2007; Porges, Doussard-Roosevelt, Portales, \& Greenspan, 1996, Calkins, 1997; Hastings et al., in press). Two parameters of RSA are typically reported: baseline RSA and RSA reactivity during challenges.

Baseline RSA is assumed to reflect resting levels of parasympathetic activity via myelinated vagal pathways originating in the brainstem (i.e., nucleus ambiguus). According to the Polyvagal Theory, high baseline RSA would index an autonomic state that fosters visceral needs in the absence of external challenge. Therefore, baseline RSA is theorized to represent an individual's stress vulnerability as well as the ability to sustain attention, engage in social communication, and regulate emotion (see Beauchanie et al., 2007; Calkins, 1997; Hastings et al., in press; Porges, 1992).

RSA during challenge conditions, or changes in RSA from baseline relative to challenge, are often used to reflect emotional responding to a variety of situations/stimuli (e.g., Graziano, Keane, \& Calkins, 2007; Porges et al., 1996). The vagal input to the heart via myelinated pathways functions like a "brake" (i.e., vagal brake) that can be engaged or disengaged to produce rapid changes in cardiovascular output to meet environmental demands (Porges et al., 1996). During stressful or challenging situations release of the vagal brake (i.e., RSA suppression or decrease in RSA) allows heart rate to rise and does not impede or compete with subsequent sympathetic influences to the heart. Such rises in heart are thought to contribute to "mobilization" of physiological resources for fight/flight behaviors supported by sympathetic nervous system activation (i.e., mobilization system, Porges, 2007, 1995). Further, during social engagement, decreases in RSA support the allocation of physiological resources toward increased neural regulation (via cranial nerves) of the striated muscles of the face and head to promote facial expression, vocalization, and listening (i.e., social communication system, Porges, 2007; Porges et al., 1996). Thus, the systematic vagal withdrawal (i.e., indexed by RSA) modulates cardiac output to match environmental demands without requiring sympathetic activation and enables the individual to maintain more optimal emotion regulation (see Porges, 2007 for review).

Measures of baseline RSA and RSA responses to challenge appear to be both fairly stable over time and predictive of risk for behavior problems for preschool-age children (e.g., Calkins \& Keane, 2004; Doussard-Roosevelt, Montgomery, Porges, 2003; Porges et al., 1996). Children with higher baseline RSA display more positive affect (Calkins, 1997), are at lower risk for behavior problems (Calkins \& Dedmon, 2000), and display higher social competence (Blair \& Peters, 2003). In addition, preschool-age children with greater RSA suppression (i.e., greater decrease of RSA) in response to challenge are less emotionally 
negative, exhibit fewer behavior problems, and have better social skills (Calkins \& Keane, 2004). Children with greater RSA suppression also exhibit more behavioral emotion regulation (Calkins, 1997; Gentzler et al., 2009), and are at lower risk for externalizing behavior problems (Calkins \& Dedmon, 2000; Calkins, Graziano, \& Keane, 2007).

Furthermore, significant decreases of RSA (i.e., greater RSA suppression) in children has been reported in response to cognitive, attentional, physical, behavioral, emotional, and social challenges (Bar-Haim, Fox, VanMeenen, \& Marshall, 2004; Gilissen, Koolstra, Ijzendoom, Bakermans-Kraneenburg, \& van der Veer, 2007; Gentzler, Santucci, Kovacs, \& Fox, 2009; Heilman et al., 2008; Suess, Porges, Plude, 1994; Weber, ver der Molen, \& Molenaar, 1994). For example, Bar-Haim et al. (2004) found that 7-year-old children who displayed a greater decrease of RSA in response to emotionally and cognitively challenging narrative tasks produced more coherent and adaptive narratives. In addition, decreases of 3and 4-year-old children's RSA have been reported in response to fear-inducing film stimuli (Gilissen et al., 2007), physical challenge (Heilman et al., 2008), and sustained attention tasks (Suess et al., 1994; Weber et al., 1994). Dale, O'Hara, Keen, and Porges (2011) reported that infants who display less suppression of RSA exhibit more high-level withdrawal responses, including verbal and physical protests, consistent with the relation hypothesized by Porges et al. (1996) between physiological regulation of the vagal brake and positive social behavior (see Porges et al., 1996). Additionally, Heilman et al. (2008) found that 3- to 4-year-old children's RSA increased during a challenging social situation (i.e., hearing evaluations from a stranger with no parent present), a response that may dampen social engagement.

1.2.3. Skin conductance: an index of emotional reactivity-Skin conductance, a measure of electrodermal activity dependent on activation of sweat glands (Boucsein, 1992), is exclusively regulated by the sympathetic nervous system. Skin conductance level (SCL), provides a sympathetic complement to the parasympathetic index, RSA. Similar to RSA, two measures of SCL are often reported: baseline SCL values and changes in SCL in response to challenge. Thus, concurrent measurement of SCL and RSA has potential for providing a more comprehensive indicator of the dynamic adjustment of sympathetic and parasympathetic activity in response to an emotional challenge.

Baseline values of SCL index resting levels of sympathetic nervous system activity. Changes in SCL index sympathetic activation in response to challenge or stressful conditions when physiological resources are shifted toward fight/flight behaviors (Boucsein, 1992). Based on the Polyvagal perspective, individuals with chronic high levels of sympathetic activity (and low levels of parasympathetic activity) are hypothesized to have the greatest stress vulnerability (Porges, 2007). Further, increases in sympathetic activity during challenge or stressful conditions are a commonly used psychophysiological index of emotional reactivity. Increases in SCL have been associated with emotional reactivity, fear and stress (e.g., Cole, Zahn-Waxler, Fox, Usher, \& Welsh, 1996; Fabes, Eisenberg, Karbon, Bernzweig, \& Speer, 1994; Fowles, Kochanska, \& Murray, 2000; Gilissen et al., 2007).

Cole et al. (1996) found that preschool-age children who displayed "modulated" emotional expression (i.e., responsivity without intense display) exhibited lower baseline SCL 
compared to children who were highly expressive (negative emotion) or inexpressive (no emotion displayed). In addition, they reported that highly expressive children displayed greater increases in skin conductance than inexpressive children during negative mood induction. Similarly, Gilissen et al. (2007) found that temperamentally fearful 4-year-old children with less harmonious relationships with their parents had greater SCL increase in response to fear-inducing film clips than fearful children with more harmonious parental relationships. Furthermore, Fabes et al. (1994) reported that for kindergarten children skin conductance was positively related to facial distress and inversely related to helpfulness. These results are consistent with Fowles et al. (2000), who found that temperament dimensions of fearfulness and effortful (or inhibitory) control were positively correlated with SCL for 4-year-old children.

1.2.4. Concurrent parasympathetic and sympathetic activity-Beauchaine (2001) suggested that sympathetic and parasympathetic activity should be considered simultaneously in order to better understand the integrated functioning of the autonomic nervous system with behavioral and psychological processes. For example, El-Sheik et al. (2009) concurrently studied SCL and RSA in children. They reported that reciprocal autonomic nervous system activity (e.g., parasympathetic decrease concurrent with sympathetic increase) was a protective factor in predicting externalizing behavior problems in the context of marital conflict, whereas opposing autonomic activity (e.g., increase of both parasympathetic and sympathetic activity) operated as a vulnerability factor. These findings are consistent with a Polyvagal perspective (see Porges, 2007), which would hypothesize that complimentary inhibition of vagal influence to the heart and sympathetic excitation to the sweat glands would provide a physiological state that would support the adaptive functions of fight/flight behaviors and that this reactivity could be immediately down regulated by a re-engagement of the vagal brake. The above findings point out the feasibility and importance of concurrently studying RSA and SCL at baseline and in response to stress when attempting to provide a more comprehensive account of psychophysiological features of childhood stuttering.

\subsection{The Present Study}

The present study assessed between-group differences in preschool-age children's psychophysiological processes during two tasks: (1) listening-viewing of emotionallyarousing video clips and (2) speaking during narrative tasks subsequent to the video clips. We assessed: (1) RSA as an index of parasympathetic activity, and (2) SCL as an index of sympathetic activity, with these indices evaluated separately as well as concurrently for both preschool-age CWS and their CWNS peers.

It was hypothesized that the baseline measures of RSA and SCL would be related to the group differences frequently observed in reactivity (e.g., Eggers et al., 2010; Karrass et al., 2006) and regulatory skills (e.g., Anderson et al., 2003; Felsenfeld et al., 2010). Similarly, it was hypothesized that measures of RSA and SCL in response to challenge and speaking tasks would be related to reported group differences in adaptive responding to challenging emotional situations (e.g., Johnson et al., 2010; Ntourou et al., 2013; Schwenk et al., 2007). If data support these hypotheses, there would be further empirical support that various 
processes associated with emotion, including autonomic state and reactivity, are associated with childhood stuttering (Conture \& Walden, 2012). Employing the Polyvagal Theory as a theoretical framework, and previous empirical findings as a guide, we empirically assessed the possibility that physiological reactions to emotion stimuli (as indexed by measures of RSA and SCL), would be associated with childhood stuttering.

In summary, the Polyvagal Theory (Porges, 2007) suggests that at baseline, high parasympathetic and low sympathetic activity is associated with better emotion regulation, social engagement, and various other adaptive outcomes. Moreover, the Polyvagal Theory emphasizes that optimal adaptive reactions during conditions of stress and challenge, are characterized by a disinhibition of the vagal brake that is characterized by a reduction in (or suppression of) RSA. Similar predictions of increases in sympathetic activity and decreases in RSA with stimuli similar to those used in the present study have been reported during emotional challenge (e.g., fear-inducing film stimuli, Gilissen et al., 2007) and narrative tasks (Bar-Haim et al., 2004). Specifically, we addressed four related issues.

First, given findings that preschool-age CWS, compared to CWNS, exhibit greater emotional reactivity and poorer emotional regulatory skills on caregiver-based reports (e.g., Karrass et al., 2006) and during behavioral observations (e.g., Arnold et al., 2011), we hypothesized that during baseline, CWS would exhibit lower RSA and greater SCL.

Second, based on findings that preschool-age CWS, compared to CWNS, exhibit increased negative affect during emotionally-arousing conditions (Johnson et al., 2010; Ntourou et al., 2013), we hypothesized that during emotionally-arousing (positive and negative) listeningviewing conditions, CWS would exhibit lower RSA and greater SCL.

Third, based on reasoning similar to that of our second hypothesis, we hypothesized that during the speaking tasks that followed the emotionally-arousing conditions, CWS would have lower RSA and greater SCL than CWNS.

Fourth, given the difficulties that CWS children have with establishing normally fluent speech (Clark, Conture, Frankel, \& Walden, 2013), we hypothesized that preschool-age CWS would exhibit lower RSA and greater SCL during both types of challenging experimental conditions (i.e., listening-viewing and speaking), with these differences being more marked during the speaking than listening-viewing tasks.

For each of the four hypotheses, we tested between-group differences in the relation between concurrent RSA and SCL activity. Based on the Polyvagal Theory (Porges, 2007), we conceptualized children's adaptive response patterns as characterized by a negative relation between sympathetic and parasympathetic activity (i.e., decrease in RSA associated with increase in SCL) and predicted that CWS would exhibit less adaptive patterns of concurrent autonomic activity than CWNS. 


\section{Methods}

\subsection{Participants}

Participants included 20 preschool-age CWS (15 male) and 21 preschool-age CWNS (11 male) between the ages of 37 and 71 months. The chronological age for CWS ( $M=48.55$, $S D=10.84)$ did not significantly differ from CWNS $(M=48.10, S D=10.17)$.

Participants' race was obtained via caregiver interview. CWS were 18 Caucasians, one African-American, and one with more than one reported race. CWNS were 18 Caucasians, and 3 with more than one reported race.

From an initial pool of 54 participants (27 CWS, 26 CWNS, 1 unclassifiable) for whom data was collected, one was excluded because talker group classification was undeterminable. Twelve other participants (7 CWS and 5 CWNS) were excluded because it was not possible to acquire RSA and/or SCL data due to non-compliance and/or excessive data artifact.

All participants were paid volunteers naïve to the purposes and methods of the study and the majority of them were recruited as part of a longitudinal study investigating the relation between stuttering and emotions conducted by Vanderbilt University's Developmental Stuttering Project. Participants were referred to the Vanderbilt Bill Wilkerson Center for participation by their parents who were informed via (a) free, widely read parent-oriented magazine, (b) local health care providers, or (c) self/professional referrals to the Vanderbilt Bill Wilkerson Hearing and Speech Center

The Vanderbilt University IRB approved the protocol. Informed consent by parents and assent by children was obtained.

\subsection{Classification and Inclusion Criteria}

Children classified as CWS exhibited 3 or more stuttered disfluencies (i.e., sound syllable repetitions, monosyllabic whole-word repetitions ${ }^{1}$ and sound prolongations) per 100 words of conversational speech and received an overall score of 11 or higher on the Stuttering Severity Instrument (SSI-3; Riley, 1994). Children classified as CWNS exhibited 2 or fewer stuttered disfluenices per 100 words of conversational speech and received an overall score of 10 or below on the SSI-3. No CWS child received or was receiving treatment for stuttering.

All participants scored at or above the $16^{\text {th }}$ percentile on standardized speech and language tests, including the Goldman Fristoe Test of Articulation (GFTA; Goldman \& Fristoe,

\footnotetext{
${ }_{1}$ Monosyllabic whole-word repetitions produced "without tension are not counted as stuttering. Repetition of one-syllable words may be stuttering if the word sounds abnormal (shortened, prolonged, staccato, tense, etc.); however, when these single-syllable words are repeated but are otherwise spoken normally, they do not qualify as stuttering using the definition just stated" (Riley, 1994, p. 4). Therefore, in accordance with the SSI-3 manual, only those monosyllabic whole-word repetitions that were perceptually "abnormal (shortened, prolonged, staccato, tense, etc.)" were included as stuttered disfluencies. In the present study, speech disfluencies that were perceptually effortless, non-tense repetitions of monosyllabic whole-words-such as those produced for emphasis (e.g., the child says, "it was a fast, fast car," while gesturing how fast the car moved)-were not counted as stuttered or nonstuttered disfluencies. These effortful, non- tense repetitions of single-syllable whole words were excluded from the disfluency data for talker group classification. It should be noted that other stuttering classification schemes-some of which exclude monosyllabic whole-word repetitions from the stuttered disfluency category-have been used particularly when assessing older, school-age and adults who stutter (e.g., Howell, Bailey, \& Kothari, 2010; Jiang, Lu, Peng, Zhu, Howell, 2012).
} 
2000), Peabody Picture Vocabulary Test (PPVT-III; Dunn \& Dunn, 1997), the Expressive Vocabulary Test (EVT-2; Williams, 1997), and the Test of Early Language Development (TELD-3; Hresko, Reid, Hammill, \& Pro-Ed (Firm), 1999). Socioeconomic status was determined by scoring parent education and occupation levels on the Hollingshead Index (1975). One-way ANOA indicated no significant differences between the two talker groups on standardized measures of speech-language abilities or social economic status (SES).

\subsection{Procedure}

Upon arrival at the Vanderbilt Developmental Stuttering Laboratory, participants were seated in a car safety seat directly in front of a computer monitor. After allowing a few minutes to acclimate, the participant's skin was cleaned with an alcohol pad to optimize electrical conductance of electrodes. Hypoallergenic electrodes were applied to the skin surface at the suprasternal notch of the rib cage and at the 12th rib laterally on the left side for acquisition of heart rate, and on the volar surfaces of the distal phalanges of the index and ring fingers of the right hand for acquisition of skin conductance. Finally, a lapel microphone was attached to the participant's clothing to acquire the audio signal.

For the pre- and post-experimental baseline conditions, participants viewed an animated screensaver of a 3-dimensional fish tank for four minutes. The screensaver contained minimal action and was assumed to be suitable to establish baseline levels of RSA and SCL activity. The screensaver was viewed once at the beginning and again at end of the experiment to obtain pre- and post-experimental baselines measures. The four minute duration of the pre- and post-baseline stimuli (i.e., fish tank screensaver) was utilized in order to control the content and duration of stimuli for all the preschool-age participants and was consistent with previous studies assessing baseline values of SCL (e.g., Cole et al., 1996; Gilissen et al., 2007) and RSA (e.g., Bar-Haim et al., 2004; Gilissen et al., 2007).

After the pre-experimental baseline, participants viewed negative and positive 4-minute audio/video clips from one of five movies appropriate for children, including Happy Feet, The Lion King, The Little Mermaid, The Wizard of Oz, and The Princess and the Frog. The video clips were intended to elicit negative and positive emotional states, and order of presentation was counterbalanced across children. After the first baseline and after each of the two film clips, participants were asked to tell a story about one of three storybooks about a boy, a dog, and a frog by the author Mercer Mayer.

The effectiveness of the audio/video clips in eliciting the expected positive, negative, and neutral emotions were validated by trained research personnel (1 PhD student and 1 Masters level research assistant). These personnel, blind to the experimental condition, viewed the video recordings of 16 preschool-age CWS $(n=8)$ and CWNS $(n=8)$ children during each of the four experimental conditions (pre-baseline, post-baseline, negative, positive) and made a judgment as to which condition the children were viewing. The research personnel accurately identified the valence of the video (baseline, positive, negative) for $78.9 \%$ of the experimental conditions. Thus, it was assumed that the experimental conditions had differential effects specific to each emotion condition and these differences were observable by judges' blind to condition. In part, we choose this approach (behavioral observation) to 
assess emotional responses rather than self-report because some children have difficulty reliably reporting on their emotional states at this age.

2.3.1. Processing of respiratory sinus arrhythmia (RSA)-To obtain RSA, an electrocardiogram (ECG) was acquired for each participant using the Biopac MP150 system (Biopac Systems, Inc.) and digitized at $1250 \mathrm{~Hz}$. The raw ECG was first band-pass filtered to remove high frequency noise and low frequency drift (high pass cutoff: $0.5 \mathrm{~Hz}$; low pass cutoff: $35 \mathrm{~Hz}$ ). Biopac software processed the ECG signal by detecting the peak of the Rwave and timing the sequential inter-beat-intervals (IBI) in ms. Inter-beat-interval time series were produced from segments within the ECG corresponding to each baseline, the negative and positive film clips, and the first four minutes of all three narrative tasks.

The IBI time series were processed by CardioEdit software (Brain-Body Center, University of Illinois at Chicago, 2007) to correct artifacts due to ventricular arrhythmias and faulty detections due to movement within the IBI series by adding, dividing, or averaging consecutive points to be consistent with surrounding points. RSA is an atrial rhythm that will be distorted when ventricular rhythms are present in the IBI time series. No more than $5 \%$ of the total data for any condition was corrected using CardioEdit. CardioBatch software (Brain-Body Center, University of Illinois at Chicago, 2007) was used to derive measures of RSA from corrected IBI files. RSA was calculated using Porges' method (Porges, 1985; Porges \& Bohrer, 1990), which uses a 21-point polynomial to detrend periodicities in the IBI series slower than RSA (e.g., basic metabolic processes often associated with vasomotor and blood pressure oscillation rather than RSA). A band-pass filter was then applied to the time-sampled IBI series (i.e., $250 \mathrm{msec}$ intervals) to extract the variance at the frequency of respiration for young children $(0.24-1.04 \mathrm{~Hz})$. Respiratory sinus arrhythmia (RSA) was expressed as the natural $\log$ of this variance: $\ln (\mathrm{ms})^{2}$ (for further details, see Lewis et al., 2012). Values of RSA were derived for sequential 30-sec epochs within each 4-minute condition (i.e, pre-baseline, negative listening-viewing task, positive listening-viewing task, three narrative speaking tasks, and post-baseline). The average of the 30 -sec epochs within each 4-minute epoch was used as the metric for RSA in the data analyses.

2.3.2. Processing of Skin Conductance Level (SCL)-The present study used mean SCL to index sympathetic activation rather than other measures of skin conductance. The measures of sympathetic and parasympathetic activity were quantified into metrics as comparable as possible. Since the mean of the 30-sec epochs within each 4- minute condition was used as RSA metric, mean skin conductance during each 4-minute condition was used as the SCL metric.

The SCL signal was acquired using the Biopac MP150 system (Biopac Systems, Inc.) and digitized at $1250 \mathrm{~Hz}$. The "Connect Endpoints" math function of Biopac Acknowledge 4.1 software was then used to correct missing data artifacts due to removal of the electrodes during data collection. No more than $5 \%$ of the total data for any epoch was corrected. Text files were then created for each epoch of SCL data, and the data were then downsampled to $125 \mathrm{~Hz}$ by retaining every $10^{\text {th }}$ data point for each epoch. From the downsampled data, a mean SCL value for the epoch was derived (after phasic responses were removed from the 
signal) and expressed in microSiemens ( $\mu \mathrm{S})$. Values of SCL were derived for sequential 30sec epochs within each 4-minute condition.

2.3.3. Measurement reliability for dependent variables-To assess measurement reliability, $20 \%$ of the total final data corpus was randomly selected to determine inter-rater reliability between the first author and trained researchers (graduate-level or above) for the measures of (a) RSA and (b) SCL.

Values of RSA for pairs of raters were within $0.10 \ln (\mathrm{ms})^{2}$ for listening-viewing (i.e., including pre- and post-baseline, negative and positive film clips) and speaking (i.e., including all three narrative tasks) tasks, with correlations of RSA values between the two researchers exceeding 0.99 for both.

A second researcher independently derived values of SCL. Values were within 0.10 microSiemens $(\mu \mathrm{S})$ for all listening-viewing and speaking tasks. Inter-judge correlations exceeded 0.99 for both.

\subsection{Data Analysis}

2.4.1. Statistical modeling - Linear mixed-effects models (LMM, Pinheiro \& Bates, 2000) were used to examine each of the four hypotheses. The LMM can be thought of as an ANOVA that better controls for correlated errors that occur with repeated measurements of an individual (Nich \& Carroll, 1997). For an example of this analytical model applied to a data set with multiple levels (i.e., repeated measurements nested within and between participants) see Key, Lambert, Aschner, and Maitre (2012). Linear mixed-effects models are often used to assess outcomes over multiple observations when time is of interest. In the present study no hypotheses were proposed to assess change over time. The LMM provide a more precise assessment of our a priori hypotheses, namely, between-group differences in RSA and SCL during various experimental conditions.

The LMMs were run using Statistical Package for the Social Sciences Statistics version 20 (SPSS Statistics). Eight separate models ${ }^{2}$ were constructed to examine the dependent measures (i.e., RSA, SCL) across the seven conditions (i.e., pre- and post-baselines, two film clips, and three narrative tasks) and between the two talker groups (i.e., CWS and CWNS). The values derived from each 4-minute condition were used as repeated measures in RSA and SCL models to assess between-group differences.

2.4.2. Fixed factors and continuous covariates-Statistical models included fixed factors and continuous covariates reflecting potential alternative explanations for the observed behavior of RSA and SCL. Four fixed factors were covaried in each RSA and SCL model: (1) condition (pre/post-baseline, negative/positive videos for listening-viewing; prebaseline/negative/positive prior manipulations for speaking), (2) film (The Lion King, The Wizard of OZ, The Little Mermaid, The princess and the frog, or Happy Feet), (3) order (positive film first vs. negative film first), and (4) gender.

${ }^{2}$ Four models of RSA and four models of SC were used to assess the theoretically-driven hypotheses. 
In each RSA model, age in months, body mass index (BMI), and an estimate of respiration ${ }^{3}$ rate (known to be related to RSA, El-Sheikh, 2005; El-Sheikh, Erath \& Keller, 2007; Reilly \& Moore, 2003) were used as covariates. To assess the impact of talking on the dependent variables, number of utterances was covaried in each of the speaking models. Similar to the guidelines for the Systematic Analysis of Language Transcripts (SALT; Miller \& Iglesias, 2008), utterance segmentation was based on either 1) a new independent clause or 2) a pause $>1$ second. Number of utterances ${ }^{4}$ was used because it has been shown to be associated with inspiratory volume and respiratory cycle (Winkworth, Davis, Adams, \& Ellis, 1995), which are related to RSA. Furthermore, in each RSA model, SCL was entered as a covariate (to evaluate the relation between RSA and SCL) along with the interaction of group and SCL (to evaluate whether the relation between RSA and SCL differed between groups). In the statistical models used to assess respiratory sinus arrhythmia (RSA) and skin conductance level (SCL) during listening-viewing and speaking conditions, baseline values of RSA and SCL were included as covariates to account for the initial value of those variables (law of initial values; Wilder, 1962). For each term in the model, Cohen's $d$, a measure of effect size, was calculated using approximate effect sizes from Type $\mathrm{III}^{5} \mathrm{~F}$-ratios based on meta-analytic formulas (Rosenthal \& DiMatteo, 2001).

\section{Results}

\subsection{Descriptive Information}

3.1.1. Speech fluency-Group differences in speech disfluency were evaluated with ANOVA. There was statistically significant difference in stuttered disfluencies per 100 words between CWS $(M=8.99, S D=5.43)$ and CWNS $(M=1.08, S D=0.78), F(1,39)=$ $43.69, p<.001, \eta^{2}=.528$, and a difference in SSI-3 scores between CWS $(M=18.50, S D=$ 5.84) and CWNS $(M=6.29, S D=1.71), F(1,39)=84.51, p<.001, \eta^{2}=.684$. In addition, there was a significant difference between CWS $(M=13.53, S D=4.47)$ and CWNS $(M=$ $5.05, S D=2.88), F(1,39)=52.65, p<.001, \eta^{2}=.574$ in total disfluencies per 100 words. However, there was no difference between CWS $(M=4.54, S D=2.97)$ and CWNS $(M=$ $3.97, S D=2.74)$ in non-stuttered disfluencies per 100 words.

\subsection{Autonomic responses}

Results pertaining to the four a priori hypotheses are presented below. Descriptive RSA and SCL data for each hypothesis are in Table 2. Results of the LMMs pertaining to the a priori hypotheses are in Table 3.

\subsubsection{Skin Conductance Level and Respiratory Sinus Arrhythmia during Baseline (Hypothesis 1)-Inconsistent with the prediction that CWS would exhibit}

\footnotetext{
${ }^{3}$ A custom program, RespFreqFromRSA (Lewis, 2010), developed in MATLAB (r2012a) was used to extract the respiration rate from the frequency of the RSA rhythm. It has previously been demonstrated that the correlation between the dominant frequency in the RSA component of the heart period time series and the spontaneous breathing rate approaches unity (Denver, Reed, \& Porges, 2007). ${ }^{4}$ It remains an open empirical question whether number of words, another possible means for measuring amount of speech, is more suitable.

${ }^{5}$ Type III tests examine the significance of each effect simultaneously (rather than sequentially) with all other effects in the model (Littell, Milliken et al., 2006). According to Cohen's (1992) guidelines, Cohen's $d$ effect size values of .2/.5/.8 may be considered small/medium/large.
} 
greater SCL during baseline, there was no significant between-group difference in SCL during either pre- or post-baseline conditions. However, consistent with prediction (see Figure 1), during the baseline conditions CWS (estimated marginal mean, $E M M=5.952$, standard error, $S E=.184)$ had significantly lower RSA than CWNS $(E M M=6.477, S E=$. 166), $F(1,46.75)=4.45, p=.040, d=.62$. During baseline there was no significant correlation between SCL and RSA, nor were the correlations different in the CWS and CWNS groups

\subsubsection{Skin Conductance Level and Respiratory Sinus Arrhythmia during} Listening-Viewing (Hypothesis 2)-Consistent with predictions that the CWS group would differ from CWNS group in SCL and RSA during listening-viewing conditions (Hypothesis 2), there was a significant group by condition interaction for SCL during the positive and negative emotionally-arousing listening-viewing conditions, $F(1,574.35)=$ $5.033, p=.025, d=.19$. Descriptively, CWS had greater SCL during the positive listeningviewing condition, whereas CWNS had greater SCL during the negative listening-viewing condition. CWS were predicted to exhibit lower RSA during listening-viewing, but there was no significant between-group difference for RSA. There was neither a significant correlation between SCL and RSA nor a difference in correlations between CWS and CWNS during listening-viewing conditions.

\subsubsection{Skin Conductance Level and Respiratory Sinus Arrhythmia during Speaking (Hypothesis 3)-Consistent with predictions that CWS would differ from} CWNS in SCL and RSA during speaking conditions (Hypothesis 3), results indicated that CWS $(E M M=18.449, S E=1.323)$, compared to CWNS $(E M M=14.576, S E=1.164)$, had higher SCL during the speaking conditions, $F(1,20.89)=4.68, p=.042, d=.95$. Furthermore, a group by speaking condition interaction (preceding listening-viewing condition: pre-baseline, negative, positive) was found, $F(2,580.73)=13.06, p<.001, d=$. 30. As shown in Figure 2, CWS had significantly higher SCL when speaking following the positive listening-viewing condition than CWNS (mean difference $=5.798, S E=1.839, \mathrm{p}=$. 004). There were no group differences when speaking following negative or neutral conditions.

During speaking conditions CWS $(E M M=5.686, S E=.100)$ had significantly lower RSA than CWNS $(E M M=5.539, S E=.082), F(1,133.97)=4.657, p=.033, d=.37$ (see Figure $3)$. There was no interaction of group and prior listening-viewing condition (pre-baseline, negative, positive). However, as shown in Figure 4, during speaking there was a betweengroup difference in the relation between RSA and SCL (interaction of group and SCL in the RSA model $), F(1,139.76)=9.060, p=.003, d=.51$. During speaking CWS had a significant positive relation between RSA and SCL (est. $\bullet=.037, \mathrm{p}<.001$ ), whereas for CWNS there was no significant relation.

\subsubsection{Physiological Response during Listening-Viewing versus Speaking} Conditions (Hypothesis 4)-Consistent with predictions that CWS would differ from CWNS in SCL and RSA during listening-viewing versus speaking conditions (Hypothesis 4), there was a significant interaction of group by task (listening-viewing vs. speaking) for SCL, $F(1,1190.30)=21.29, p<.001, d=.27$. As shown in Figure 5, CWS, when compared 
to CWNS, had greater SCL during listening-viewing (mean difference $=1.674, S E=.516, \mathrm{p}$ $=.001)$, but there was no difference between the groups during speaking.

As shown in Figure 6, there was an interaction of group and task (listening-viewing vs. speaking) for RSA, $F(1,818.05)=4.621, p=.032, d=.15$. CWS had higher RSA during listening-viewing than CWNS (mean difference $=.174, S E=.068, p=.011$ ), whereas there was no difference between the groups during speaking.

3.2.5. Summary of main findings-The present study's main findings indicated that: (1) during baseline, preschool-age CWS had significantly lower RSA than their CWNS peers (Hypothesis 1); (2) during positive listening-viewing, CWS had significantly higher SCL than CWNS (Hypothesis 2); (3) during speaking CWS had greater SCL and RSA during speaking following the positive listening-viewing condition (Hypothesis 3); (4) during speaking, CWS exhibited a significant positive relation between RSA and SCL, whereas CWNS exhibited no relation (Hypothesis 3); and (5) during listening-viewing (compared to speaking), CWS had greater SCL and RSA than CWNS (Hypothesis 4).

\section{Discussion}

The main findings will be discussed in terms of the four a priori hypotheses, which will be followed by a discussion of the clinical implications and limitations of present findings.

\subsection{A priori hypotheses}

\subsubsection{Skin Conductance Level ${ }^{6}$ and Respiratory Sinus Arrhythmia ${ }^{7}$ during Baseline (Hypothesis 1)—During baseline conditions, CWS did not differ from CWNS} in terms of sympathetic activation, as measured by SCL. They did, however, have lower parasympathetic activity as measured by RSA. The latter finding suggests that preschool-age CWS exhibit less vagal regulation of the heart, consistent with initial predictions. Consistent with the Polyvagal Theory, lower RSA during baseline increases the vulnerability to react to challenges with a sympathetic response.

Empirically, typically developing children with high baseline RSA exhibit a greater range of autonomic reactivity in response to challenge, which is paralleled by behaviors that rapidly stabilize (e.g., Calkins, 1997; Calkins \& Keane, 2004; Porges, et al., 1996). High baseline RSA has been associated with children who express more positive affect (Calkins, 1997) and are at lower risk for behavior problems (Calkins \& Dedmon, 2000). In contrast, children with difficulties regulating behaviors during social demands often have low baseline RSA (Beauchanie et al., 2007; Hastings et al., in press; Porges, et al. 1996).

In the literature, the association between individual differences in RSA and behavior regulation during early childhood has implications for understanding the behavior observed in CWS. Since CWS had lower RSA during baseline, the literature with developing children

\footnotetext{
${ }^{6}$ Skin conductance level is used to index sympathetic nervous system activity, which is often described as a physiological feature of emotional reactivity.

${ }^{7}$ Respiratory sinus arrhythmia is used to index parasympathetic activity, which is often described as a physiological feature of emotion regulation.
} 
(i.e., CWNS) suggests that a dampened vagal regulation circuit may contribute to the behavior regulation problems frequently observed in CWS. For example, CWS tend to exhibit poor regulatory skills (e.g., Anderson et al., 2003; Arnold et al., 2011; Eggers et al., 2010; Felsenfeld et al., 2010; Karrass et al., 2006; Walden et al., 2012). Eggers et al. (2010) suggested that temperamental proclivities toward increased reactivity and decreased regulation may contribute to stuttering in stressful situations. If CWS have decreased potential for emotion regulation during non-challenging, everyday situations (e.g., baseline conditions), then they may not have the "neural" resource to regulate their emotional state when challenged during speaking situations. This potential link between the regulation of behavioral and physiological state is supported in the current study.

\subsubsection{Skin Conductance Level and Respiratory Sinus Arrhythmia during Listening-Viewing (Hypothesis 2)—CWS had greater SCL during the positive} listening-viewing condition (vs. negative), whereas CWNS had greater SCL during the negative (vs. positive) condition. Although increases in preschool-age children's skin conductance have been reported during negative emotional conditions (e.g., Cole et al., 1996; 2000; Gilissen et al., 2007), others have reported increases during positive visual stimuli (Fowles et al., 2000).

Fowles et al. (2000) discussed the possibility that electrodermal (i.e., SCL) activity may be negatively biased (more responsive to negative stimuli), but that such bias may also be related to temperament. Thus, there may be specific temperamental profiles in which electrodermal activity may be more positively biased (more responsive to positive stimuli). Considering this, it is possible that temperamental characteristics of CWS may render them more likely than their peers to exhibit greater skin conductance during positive conditions. However, in the present study temperamental characteristics (e.g., negative affect, effortful control, etc., Rothbart, 2011) were not measured. Future research with CWS may investigate this possible association between electrodermal activity and temperament. The observation in present study of increased SCL during a positive listening-viewing condition could be consistent with speculation (Adams, 1992) and empirical evidence (e.g., Johnson et al., 2010; Jones et al., in press) suggesting that childhood stuttering increases when positive emotion is experienced.

\subsubsection{Skin Conductance Level and Respiratory Sinus Arrhythmia during} Speaking (Hypothesis 3)-CWS displayed greater skin conductance level during the speaking task following the positive condition than CWNS. This finding suggests that the increased arousal expressed in the CWS during the positive emotion condition was maintained during the succeeding speaking condition. There is mounting evidence that indicates a propensity for preschool-age CWS's positive emotional arousal to extend into subsequent speech and social communication activities, some of which have been associated with stuttered speech (e.g., Johnson et al., 2010; Jones et al., in press). Future empirical studies will need to investigate whether the sympathetic activation elicited during positive emotion conditions/speaking tasks is associated with stuttering (for further discussion of childhood stuttering associated with positive conditions, see Adams, 1992), an association reported with behavioral aspects of emotionality (Walden et al., 2012). 
CWNS were hypothesized to have higher RSA during speaking than CWS. However, the opposite was observed. Lower RSA for CWNS may represent a more adaptive physiological response to narrative tasks, similar to findings that children who display the greatest decrease of RSA during emotionally and cognitively challenging narrative tasks produce the most coherent and adaptive narratives (Bar-Haim et al., 2004). Infants with this pattern of decreases in RSA during social engagement challenges developed into more behaviorally regulated children (see Porges et al., 1996). In addition, Dale et al. (2011) reported that increases in RSA during social engagement challenges during infancy were a marker for severe behavior regulation difficulties during childhood. This is an intriguing result, in that it is consistent with findings that adults who stutter (AWS) have increased heart rate during speaking that is less marked than AWNS (Caruso et al., 1994; Peters \& Hulstijn, 1984; Weber \& Smith, 1990). As Alm (2004) suggested, this may indicate a "freezing response," an idea congruent with Polyvagal Theory's "mobilization system" (Porges, 2007).

From a Polyvagal perspective, freezing behaviors require increased cardiac output (i.e., increase in heart rate and reduction in RSA), with such change in output needed to support the increased muscle tone associated with behavioral "freezing" as a preparatory response to fight or flee. However, the lack of decreases in RSA for CWS, when the normative agerelated response is to reduce RSA as a preparatory response to engage, has been seen in infants who lack engagement skills and functionally dissociate from social cues (Dale et al., 2011). Similar responses have been observed in children diagnosed with autism spectrum disorders (see Porges et al., 2013; Patquin et al., 2013), who exhibit increases in RSA during tasks requiring attention to social cues. This inability to efficiently remove the vagal brake during challenge has been observed in children, diagnosed with selective mutism, who have difficulty initiating speech. Research characterizes children with selective mutism as having a sluggish vagal brake (Heilman et al., 2012). For CWS, the lack of RSA decrease observed may be a feature consistent with having a sluggish vagal brake, which may in turn influence CWS's ability to effectively and efficiently engage during social-communication situations, a possibility that must await further empirical study.

During speaking, CWS also displayed a significant positive relation between RSA and SCL, whereas there was no relation between RSA and SCL in the CWNS group. This finding supports the hypothesis that during speaking preschool-age CWS exhibit a less adaptive (i.e., oppositional) pattern of ANS activity. However, it does not support the prediction that CWNS would exhibit a more adaptive (i.e., reciprocal) pattern of sympathetic and parasympathetic activity. This interpretation was based on predictions of Polyvagal Theory (Porges, 2007) suggesting that well-regulated autonomic systems exhibit reciprocal activity, as well as empirical evidence that opposing autonomic activity (e.g., simultaneous increases in sympathetic and parasympathetic activity) predicts externalizing behavior problems and is associated with attention problems (El-Sheik et al., 2009).

Ideally, release of the vagal brake (i.e., decrease in RSA) allows for either the potential for or actual increase in sympathetic activity as required by situational demands. In contrast, oppositional activity (e.g., high RSA concurrent with high SCL) may diminish the individual's ability to rapidly and adaptively react physiologically and emotionally to dynamically changing environmental requirements. Thus, a simultaneous increase of 
parasympathetic and sympathetic activity may be associated with dysregulated responses to challenging situations (Porges, 1995, 2001). Consistent with this explanation, El-Sheik et al. (2009) reported that children with attention problems displayed patterns of opposing autonomic activity (i.e., co-activity or co-inhibition).

From an evolutionary perspective, Polyvagal Theory (Porges, 2007) suggests that a dysregulated mobilized defensive response (increased sympathetic and parasympathetic activity) or an immobilized response (decreased sympathetic and an increase in the phylogenetically older vagal pathway that supports behavioral shutdown) are both not supportive of social engagement. These response patterns might be expected during challenging situations that would be perceived as frightening. Whereas early in mammalian evolutionary history these patterns of physiological responding would likely occur in response to threats related to physical survival, challenges in the social environment can also lead to these patterns of physiological responses (e.g., Dickerson \& Kemeny, 2004).

In the current study, we speculate that CWS may react to the narrative tasks as more challenging than CWNS. This increase in negative valence of the task might be related to prior experiences with speaking disfluently, a possibility consistent with self-reports (Clark et al., 2012). Alm noted that a "freezing response" (consistent with the defense response proposed by the Polyvagal Theory) has been observed during speech of adults who stutter. In the preschool-age CWS of the present study, it is possible that there are two patterns of responses present for subsets of children, with some CWS exhibiting a dysregulated mobilization (increased sympathetic and parasympathetic) response during speech, and others exhibiting a more passive "freezing" response (decreased sympathetic and parasympathetic). A better understanding of the potential impact of dysregulated autonomic patterns (e.g., high RSA and SCL) on the speech fluency of preschool-age CWS awaits future study.

\subsubsection{Physiological Response during Listening-Viewing versus Speaking} Conditions (Hypothesis 4)—During listening-viewing (compared to speaking tasks) CWS exhibited greater sympathetic activity (SCL) than CWNS, suggesting that they may be more reactive to environmental stimuli. This psychophysiological finding is consistent with behavioral reports that CWS are more emotionally reactive to emotional challenges (Johnson et al., 2010; Ntourou et al., 2013) and irrelevant background stimuli (Schwenk et al., 2007).

Likewise, CWS exhibited higher parasympathetic activity (RSA) during listening-viewing (compared to speaking) conditions than CWNS. Based on Polyvagal Theory (Porges, 2007, 2011) as well as empirical investigations of RSA during fear-inducing films (Gilissen et al., 2007), one adaptive pattern of autonomic nervous system responding would be decreased parasympathetic activity (decreased or suppression of RSA) during emotional challenge. Therefore, the current finding for RSA suggests that CWS have difficulty regulating physiological state during emotional challenge, consistent with caregiver reports of regulatory difficulties in this population (e.g., Karrass et al., 2006; Felsenfeld et al., 2010). 


\subsection{General Implications of Findings for Childhood Stuttering}

Overall, present findings were taken to suggest that CWS exhibit lower potential for emotion regulation (i.e., lower baseline RSA) coupled with less adaptive patterns of sympathetic and parasympathetic activity during speaking and emotional challenge. One may speculate how this pattern of physiological activity might impact the fluency of speech production in challenging communicative contexts.

First, it is worth considering that, higher baseline RSA is predictive of better performance on tasks of (1) working memory and cognitive efficiency in children aged six to thirteen years (Staton, El-Sheik, Buckhalt, 2008) and (2) executive function in preschool-age children (e.g., number recall and children's Stroop test; Marcovitch et al., 2010). Higher baseline RSA has also been associated with better social behavior and receptive language in children with autism (Patriquin Scarpa, Friedman, \& Porges, 2011). In addition, Marcovitch et al. (2010) found that children, who displayed a moderate decrease in RSA during cognitive challenge, performed better than those had diminished or exaggerated decreases in RSA. Given these findings, it is possible that the lower RSA in CWS might be associated with the memory and attention processes that support speech-language planning and production, including (a) short term memory performance implicated in phonological processing (e.g., Bajaj, 2007), (b) receptive and/or expressive language abilities (e.g., Ntourou, Conture, Lipsey, 2011) and (c) attentional processes (e.g., Eggers et al., 2012; Felsenfeld et al., 2010).

To the extent that the processes that support physiological or emotional arousal share resources with processes that support speech production (e.g., memory \& attention), speech fluency may be compromised under conditions of elevated emotional or physiological arousal. Thus, conditions that require CWS (compared to CWNS) to flexibly adapt might negatively impact speech fluency if the resources that support adaptation to change (e.g., disengaging, shifting and re-engaging attention) are also needed for speech. This would be important for children, whose memory and attention skills are still developing. Such speculation is consistent with findings that stuttering is associated with emotional (Arnold et al., 2011; Ntourou et al., 2013; Walden et al., 2012) and cognitive stress (Caruso et al., 1994). Furthermore and similar to Eggers et al.'s (2010) speculation, a proclivity toward maladaptive patterns of physiological activity during speaking may increase the likelihood that CWS would associate such situations with emotional/social challenge rather than a positive social communication opportunity, speculation consistent with that of the "experience" aspect of Conture et al.'s (2006) Communication-Emotional Model of Stuttering. Thus, consistent with the Polyvagal theory (Porges, 2001, 2007), CWS would react with a physiology that would support defense (i.e., fight/flight or freeze), rather than a physiology that would support reciprocal social engagement behaviors.

Perhaps for CWS, particularly those who tend to be more behaviorally inhibited (Choi et al., 2013), novel social contexts bring about anticipated uncertainty to which they respond with elevated sympathetic and parasympathetic arousal (similar to Alm's (2004) notion of sympathetic and parasympathetic "co-activation"). Anticipated uncertainty associated within novel social contexts is also consistent with recurring empirical findings that young CWS exhibit poor adaptability (for review, see Conture, Kelly, \& Walden, 2013). These intriguing possibilities await further empirical study. 


\subsection{Limitations}

The present study assessed SCL and RSA during a speaking task, but did not assess the association between these physiological measures and instances of stuttering. Thus, the possible relation of these physiological behaviors and specific occurrences of stuttered speech in preschool-age children is an issue that awaits future empirical study.

The present study attempted to account for possible influences of amount of talking on respiration and RSA. Presently, however, the best means for taking into account the impact of speech-related respiration on RSA remains unclear and there is a need for further study to determine if speaking (and other activity) distorts the quantification or interpretation of RSA.

\section{Conclusion}

The present findings provide a physiological "perspective" to interpret earlier empirical investigations of emotion reactivity and childhood stuttering based on caregiver-report questionnaires (e.g., Anderson et al., 2003; Eggers et al., 2010; Felsenfeld et al., 2010; Karrass et al., 2006) and behavioral observations (Arnold, et al., 2011; Eggers et al., 2012; Johnson et al., 2010; Schwenk et al., 2007; Walden et al., 2012). Consistent with previous studies, the present physiological findings indicate that emotional processes differ between preschool-age CWS and their CWNS peers. Based on the present findings CWS, when compared to fluent peers, have greater stress vulnerability (lower parasympathetic tone during baseline), as well as greater mobilization of resources in support of emotional reactivity (more sympathetic activity during positive conditions). This pattern of response may divert CWS's resources away from attentional, cognitive and speech-language processes needed to fluently initiate and/or maintain communication. The empirical search for answers to either possibility is important to a comprehensive understanding of childhood stuttering.

It is likely that the reported group differences in emotional processes represent a complex interleaving of genetic (Rothbart, 2007; Saudino, 2005) and experiential/environmental (Goldsmith et al., 1987) influences. While these findings may reflect a "rebounding effect" due to these children's, although relatively limited, experience with or reaction to stuttering (Treon, 2010), differences in emotional processes for preschool-age CWS are not likely to be solely caused by experience with stuttering. Thus, while reducing stuttering to a pure physiological process is unwarranted, the present findings suggest that parasympathetic and sympathetic nervous system activity is involved.

\section{Acknowledgments}

This research was supported in part by National Institutes of Health (NIH) grants from the National Institute on Deafness and Other Communication Disorders (NIDCD) to Vanderbilt University (5R01DC000523-17), the National Center for Research Resources, a CTSA grant (1 UL1 RR024975-01) to Vanderbilt University that is now at the National Center for Advancing Translational Sciences (UL1 TR000445-06). The research reported herein does not reflect the views of the NIH, NIDCD, or Vanderbilt University. 


\section{References}

Adams M. Childhood stuttering under "positive" conditions. American Journal of Speech-Language Pathology. 1992; 1:5-6.

Alm PA. Stuttering, emotions, and heart rate during anticipatory anxiety: a critical review. Journal of Fluency Disorders. 2004; 29:123-33. [PubMed: 15178128]

Alm PA, Risberg J. Stuttering in adults: the acoustic startle response, temperamental traits, and biological factors. Journal of Communication Disorders. 2007; 40:1-41. [PubMed: 16814317]

Anderson JD, Pellowski MW, Conture EG, Kelly EM. Temperamental characteristics of young children who stutter. Journal of Speech Language and Hearing Research. 2003; 46(5):1221-1233.

Arnold HS, Conture EG, Key AP, Walden T. Emotional reactivity, regulation and childhood stuttering: A behavioral and electrophysiological study. Journal of communication disorders. 2011; 44(3):276293. [PubMed: 21276977]

Bajaj A. Working memory involvement in stuttering: exploring the evidence and research implications. Journal of Fluency Disorders. 2007; 32:218-38. [PubMed: 17825670]

Beauchaine T. Vagal tone, development, and Gray's motivational theory: Toward an integrated model of autonomic nervous system functioning in psychopathology. Development and Psychopathology. 2001; 13:183-214. [PubMed: 11393643]

Beauchaine TP, Gatzke-Kopp L, Mead HK. Polyvagal Theory and developmental psychopathology: Emotion dysregulation and conduct problems from preschool to adolescence. Biological Psychology. 2007; 74(2):174-184. [PubMed: 17045726]

Berntson GG, Cacioppo JT, Grossman P. Whither vagal tone. Biological Psychology. 2007; 74(2): 295-300. [PubMed: 17046142]

Blair C, Peters R. Physiological and neurocognitive correlates of adaptive behavior in preschool among children in head start. Developmental Neuropsychology. 2003; 24(1):479-497. [PubMed: 12850755]

Bosshardt HG. Cognitive processing load as a determinant of stuttering: summary of a research programme. Clinical Linguistics \& Phonetics. 2006; 20:371-85. [PubMed: 16728334]

Boucsein, W. Electrodermal activity. Plenum Press; New York: 1992.

Cabanac M. What is emotion? Behavioural Processes. 2002; 60(2):69-83. [PubMed: 12426062]

Calkins SD. Cardiac vagal tone indices of temperamental reactivity and behavioral regulation in young children. Developmental Psychobiology. 1997; 31:125-135. [PubMed: 9298638]

Calkins SD, Dedmon SA. Physiological and behavioral regulation in two-year-old children with aggressive/destructive behavior problems. Journal of Abnormal Psychology. 2000; 28:103-118.

Calkins SD, Graziano PA, Keane SP. Cardiac vagal regulation differentiates among children at risk for behavior problems. Biological psychology. 2007; 74(2):144-153. [PubMed: 17055141]

Calkins SD, Keane SP. Cardiac vagal regulation across the preschool period: stability, continuity, and implications for childhood adjustment. Developmental psychobiology. 2004; 45(3):101-112. [PubMed: 15505799]

CardioBatch. Brain-Body Center. University of Illinois at Chicago; 2007.

CardioEdit. Brain-Body Center. University of Illinois at Chicago; 2007.

Caruso AJ, Chodzko-Zajko WJ, Bidinger DA, Sommers RK. Adults who stutter: responses to cognitive stress. Journal of Speech and Hearing Research. 1994; 37(4):746-754. [PubMed: 7967559]

Carver, CS.; Scheier, MF. On the Self-Regulation of Behavior. Cambridge University Press; 2001.

Choi D, Conture EG, Walden TA, Lambert WE, Tumanova V. Behavioral inhibition and childhood stuttering. Journal of Fluency Disorders. 2013; 38(2):171-183. [PubMed: 23773669]

Clark CE, Conture EG, Frankel CB, Walden TA. Communicative and psychological dimensions of the KiddyCAT. Journal of Communication Disorders. 2012; 45(3):223-234. [PubMed: 22333753]

Cohen J. A power primer. Psychological Bulletin. 1992; 112:155-159. [PubMed: 19565683]

Cole PM, Zahn-Waxler C, Fox NA, Usher BA, Welsh JD. Individual differences in emotion regulation and behavior problems in preschool children. Journal of Abnormal Child Psychology. 1996; 105:518-529. 
Conture EG, Kelly EM, Walden TA. Temperament, speech and language: An overview. Journal of Communication Disorders. 2013; 46(2):125-142. [PubMed: 23273707]

Conture, EG.; Walden, TA. Dual Diathesis-Stressor Model of Stuttering. In: Beliakova, L.; Filatova, Y., editors. Theoretical Issues of Fluency Disorders. Vlados; Moscow: 2012. p. 94-127.

Conture, EG.; Walden, TA.; Arnold, HS.; Graham, CG.; Hartfield, KN.; Karrass, J. A communicationemotional model of stuttering. In: Bernstein Ratner, A.; Tetnowski, J., editors. Current issues in stuttering research and practice. Lawrence Erlbaum Associates, Publishers; Mahwah, NJ: 2006. p. $17-46$.

Craig A. An investigation into the relationship between anxiety and stuttering. Journal of Speech and Hearing Disorders. 1990; 55(2):290. [PubMed: 2329791]

Craig A, Hancock K, Tran Y, Craig M. Anxiety levels in people who stutter: A randomized population study. Journal of Speech, Language and Hearing Research. 2003; 46(5):1197.

Dale LP, O'Hara EA, Keen J, Porges SW. Infant regulatory disorders: Temperamental, physiological, and behavioral features. Journal of Developmental and Behavioral Pediatrics. 2011; 32(3):216224. [PubMed: 21057324]

Denver JW, Reed SF, Porges SW. Methodological issues in the quantification of respiratory sinus arrhythmia. Biological Psychology. 2007; 74:286-294. [PubMed: 17067734]

Dietrich S, Roaman MH. Physiologic arousal and predictions of anxiety by people who stutter. Journal of Fluency Disorders. 2001; 26:207-225.

Dickerson SS, Kemeny ME. Acute stressors and cortisol responses: a theoretical integration and synthesis of laboratory research. Psychological Bulletin. 2004; 130:355-91. [PubMed: 15122924]

Doussard-Roosevelt JA, Montgomery LA, Porges SW. Short-term stability of physiological measures in kindergarten children: respiratory sinus arrhythmia, heart period, and cortisol. Developmental psychobiology. 2003; 43(3):230-242. [PubMed: 14558045]

Dunn, L.; Dunn, L. Peabody picture vocabulary test-III (PPVT-III). 3rd ed.. American Guidance Service, Inc; Circle Pines, MN: 1997.

Eggers K, De Nil LF, van Den Bergh BRH. Factorial temperament structure of stuttering, voice disordered, and normal speaking children. Journal of Speech, Language and Hearing Research. 2009; 52:1610-1622.

Eggers K, De Nil LF, Van den Bergh BR. Temperament dimensions in stuttering and typically developing children. Journal of Fluency Disorders. 2010; 35(4):355-372. [PubMed: 21130269]

Eggers K, De Nil LF, Van den Bergh BR. The efficiency of attentional networks in children who stutter. Journal of Speech, Language, and Hearing Research. 2012; 55(3):946-959.

Eggers K, De Nil LF, Van den Bergh BRH. Inhibitory control in childhood stuttering. Journal of Fluency Disorders. 2013; 38(1):1-13. [PubMed: 23540909]

El-Sheikh M. Stability of respiratory sinus arrhythmia in children and young adolescents: A longitudinal examination. Developmental Psychobiology. 2005; 46(1):66-74. [PubMed: 15690389]

El-Sheikh M, Erath SA, Keller PS. Children's sleep and adjustment: the moderating role of vagal regulation. Journal of Sleep Research. 2007; 16(4):396-405. [PubMed: 18036085]

El-Sheikh M, Kouros CD, Erath S, Cummings EM, Keller P, Staton L. Marital Conflict and Children's Externalizing Behavior: Interactions between Parasympathetic and Sympathetic Nervous System Activity. Monographs of the Society for Research in Child Development. 2009; 74(1):1. +

Fabes RA, Eisenberg N, Karbon M, Bernzweig J, Speer AL, Carlo G. Socialization of children's vicarious emotional responding and prosocial behavior: Relations with Mothers' perceptions of children's emotional reactivity. Developmental Psychology. 1994; 30(1):44-55.

Felsenfeld S, van Beijsterveldt CEM, Boomsma DI. Attentional Regulation in Young Twins With Probable Stuttering, High Nonfluency, and Typical Fluency. Journal of Speech Language and Hearing Research. 2010; 53(5):1147-1166.

Fletcher JM. An experimental study of stuttering. The American Journal of Psychology. 1914; 25:201255.

Fowles DC, Kochanska G, Murray K. Electrodermal activity and temperament in preschool children. Psychophysiology. 2000; 37:777-787. [PubMed: 11117458] 
Gentzler AL, Santucci AK, Kovacs M, Fox NA. Respiratory sinus arrhythmia reactivity predicts emotion regulation and depressive symptoms in at-risk and control children. Biological Psychology. 2009; 82:156-163. [PubMed: 19596044]

Gilissen R, Koolstra CM, van Ijzendoorn MH, Bakermans-Kranenburg MJ, van der Veer R. Physiological reactions of preschoolers to fear-inducing film clips: effects of temperamental fearfulness and quality of the parent-child relationship. Developmental psychobiology. 2007; 49(2):187-195. [PubMed: 17299791]

Goldman, R.; Fristoe, M. Goldman Fristoe 2 test of articulation. 2nd ed.. American Guidance Service; Circle Pines, MN (Publishers' Bldg., Circle Pines 55014-1796): 2000.

Goldsmith HH, Buss AH, Plomin R, Rothbart MK, Thomas A, Chess S, Hinde RA, McCall RB. Roundtable: what is temperament? Four approaches. Child Development. 1987; 58(2):505-529. [PubMed: 3829791]

Gray BB, Karmen JL. The relationship between nonverbal anxiety and stuttering adaptation. Journal of Communication Disorders. 1967; 1(2):141-151.

Graziano PA, Keane SP, Calkins SD. Cardiac vagal regulation and early peer status. Child Development. 2007; 78(1):264-278. [PubMed: 17328704]

Hastings PD, Kahle S, Nuselovici JM. How Well Socially Wary Preschoolers Fare Over Time Depends on Their Parasympathetic Regulation and Socialization. Child Development. in press.

Heilman KJ, Connolly SD, Padilla WO, Wrzosek MI, Graczyk PA, Porges SW. Sluggish vagal brake reactivity to physical exercise challenge in children with selective mutism. Development and Psychopathology. 2012; 24(01):241-250. [PubMed: 22293007]

Hollingshead, A. Four factor index of social status. Yale University; New Haven, CT: 1975.

Howell P, Bailey E, Kothari N. Changes in the pattern of stuttering over development for children who recover or persist. Clinical Linguistics \& Phonetics. 2010; 24(7):556-575. [PubMed: 20462359]

Hresko, WP.; Reid, DK.; Hammill, DD.; Pro-Ed (Firm). TELD-3 : test of early language development : examiner's manual. 3rd ed.. Pro-ed; Austin, Texas: 1999.

Izard CE. The many meanings/aspects of emotion: Definitions, functions, activation, and regulation. Emotion Review. 2010; 2(4):363-370.

Jiang J, Lu C, Peng D, Zhu C, Howell P. Classification of Types of Stuttering Symptoms Based on Brain Activity. PLoS ONE. 2012; 7(6):e39747. [PubMed: 22761887]

Johnson KN, Conture EG, Walden TA. Efficacy of attention regulation in preschool-age children who stutter: A preliminary investigation. Journal of Communication Disorders. 2012; 45(4)

Johnson KN, Walden TA, Conture EG, Karrass J. Spontaneous Regulation of Emotions in Preschool Children Who Stutter: Preliminary Findings. Journal of Speech Language and Hearing Research. 2010; 53(6):1478-1495.

Jones, R.; Choi, D.; Conture, E.; Walden, T. Seminars in Speech and Language. Temperament, emotion and childhood stuttering. in press

Jones R, Conture E, Walden T. Emotional reactivity and regulation associated with fluent and stuttered utterances of preschool-age children who do and do not stutter. Journal of Communication Disorders. in press.

Karrass J, Walden TA, Conture EG, Graham CG, Arnold HS, Hartfield KN, Schwenk KA. Relation of emotional reactivity and regulation to childhood stuttering. Journal of communication disorders. 2006; 39(6):402-423. [PubMed: 16488427]

Kefalianos E, Onslow M, Block S, Menzies R, Reilly S. Early stuttering, temperament and anxiety: Two hypotheses. Journal of Fluency Disorders. 2012; 37(3):151-163. [PubMed: 22682317]

Key APF, Lambert WE, Aschner JL, Maitre ML. Influence of gestational age and postnatal age on speech sound processing in NICU infants. Psychophysiology. 2012; 49:720-731. [PubMed: 22332725]

Lewis, G. Brain-Body Center. University of Illinois at Chicago; 2010. RespFreqFromRSA [Computer software].

Lewis G, Furman S, McCool M, Porges S. Statistical strategies to quantify respiratory sinus arrhythmia: Are commonly used metrics equivalent? Biological Psychology. 2012; 89:349-364. [PubMed: 22138367] 
Lewis KE, Golberg LL. Measurements of temperament in the identification of children who stutter. International Journal of Language \& Communication Disorders. 1997; 32(4):441-448.

Littell, RC.; Milliken, GA., et al. SAS System for Mixed Models. Second Edition. SAS Institute; Cary, N.C.: 2006.

Marcovitch S, Leigh J, Calkins SD, Leerks EM, O'Brien M, Blankson AN. Moderate vagal withdrawal in 3.5-year-old children is associated with optimal performance on executive function tasks. Developmental Psychobiology. 2010; 52(6):603-608. [PubMed: 20806334]

Miller, J.; Iglesias, A. Systematic Analysis of Language Transcipts (SALT). Version Research Version 2008. SALT Software, LLC; 2008. Computer Software

Nich C, Carroll K. Now you see it, now you don't: A comparison of traditional versus random-effects regression models in analysis of longitudinal follow-up data from a clinical trial. Journal of Consulting and Clinical Psychology. 1997; 65(2):252-261. [PubMed: 9086688]

Ntourou K, Conture EG, Lipsey MW. Language abilities of children who stutter: A meta-analytical review. American Journal of Speech-Language Pathology. 2011; 20:18.

Ntourou K, Conture EG, Walden TA. Emotional reactivity and regulation in preschool-age children who stutter. Journal of Fluency Disorders. 2013; 38(3):260-274. [PubMed: 24238388]

Patriquin MA, Scarpa A, Friedman BH, Porges SW. Respiratory sinus arrhythmia: A marker for positive social functioning and receptive language skills in children with autism spectrum disorders. Developmental Psychobiology. 2013; 55(2):101-112. [PubMed: 22212893]

Peters HFM, Hulstijn W. Stuttering and anxiety: The difference between stutterers and nonstutterers in verbal apprehension and physiologic arousal during the anticipation of speech and non-speech tasks. Journal of Fluency Disorders. 1984; 9:67-84.

Pinheiro, JC.; Bates, DM. Mixed-effects models in S and S-Plus. Springer; New York, NY: 2000.

Porges, SW. Washington DC Patent No. 4,510,944. U. S. P. Office. 1985.

Porges SW. Vagal Tone: A Physiologic Marker of Stress Vulnerability. Pediatrics. 1992; 90(3):498504. [PubMed: 1513615]

Porges SW. Orienting in a defensive world: Mammalian modifications of our evolutionary heritage. A polyvagal theory. Psychophysiology. 1995; 32:301-318. [PubMed: 7652107]

Porges SW. The polyvagal perspective. Biological Psychology. 2007; 74(2):116-143. [PubMed: 17049418]

Porges, SW. The polyvagal theory: neurophysiological foundations of emotions, attachment, communication, and self-regulation. W. W. Norton; New York: 2011.

Porges, SW.; Bohrer, RE. Analyses of periodic processes in psychophysiological research. In: Cacioppo, JT.; Tassinary, LG., editors. Principles of Psychophyiology: Physical, Social, and Inferential Elements. Cambridge University Press; New York: 1990. p. 708-753.

Porges SW, Doussard-Roosevelt JA, Portales AL, Greenspan SI. Infant regulation of the vagal "brake" predicts child behavior problems: a psychobiological model of social behavior. Developmental psychobiology. 1996; 29(8):697-712. [PubMed: 8958482]

Porges SW, Furman SA. The early development of the autonomic nervous system provides a neural platform for social behaviour: A polyvagal perspective. Infant and Child Development. 2011; 20(1):106-118. [PubMed: 21516219]

Porges SW, Macellaio M, Stanfill SD, McCue K, Lewis GF, Harden ER, Heilman KJ. Respiratory sinus arrhythmia and auditory processing in autism: Modifiable deficits of an integrated social engagement system? International Journal of Psychophysiology. 2013; 88(3):261-270. [PubMed: 23201146]

Reed CG, Lingwall JB. Conditioned stimulus effects on stuttering and GSRs. Journal of Speech and Hearing Research. 1980; 23:336-343. [PubMed: 7442194]

Reilly KJ, Moore CA. Respiratory sinus arrhythmia during speech production. Journal of Speech, Language, and Hearing Research. 2003; 46:164-77.

Riley, GD. Stuttering severity instrument for children and adults. 3rd Ed.. Pro-Ed; Austin, TX: 1994.

Rosenthal R, DiMatteo MR. Meta analysis: Recent developments in quantitive methods for literature reviews. Annual Review of Psychology. 2001; 52:59-82. 
Rothbart MK. Temperament, Development, and Personality. Current Directions in Psychological Science. 2007; 16(4):207-212.

Rothbart, MK. Becoming Who We Are: Temperament and Personality in Development. Guilford Press; 2011.

Rothbart MK, Ahadi SA, Evans DE. Temperament and personality: Origins and outcomes. Journal of Personality and Social Psychology. 2000; 78(1):122-135. [PubMed: 10653510]

Saudino KJ. Behavioral Genetics and Child Temperament. Journal of Developmental and Behavioral Pediatrics. 2005; 26(3):214-223. [PubMed: 15956873]

Schwenk KA, Conture EG, Walden TA. Reaction to background stimulation of preschool children who do and do not stutter. Journal of communication disorders. 2007; 40(2):129-141. [PubMed: 16876188]

Sheehan JG. Theory and treatment of stuttering as an approach-avoidance conflict. The Journal of Psychology. 1953; 36(1):27-49.

Sonnemans J, Frijda NH. The structure of subjective emotional intensity. Cognition \& Emotion. 1994; 8(4):329-350.

Staton L, El-Sheikh M, Buckhalt JA. Respiratory sinus arrhythmia and cognitive functioning in children. Developmental Psychobiology. 2009; 51(3):249-258. [PubMed: 19107730]

Suess PE, Porges SW, Plude DS. Cardiac vagal tone and sustained attention in school-age children. Psychophysiology. 1994; 31:17-22. [PubMed: 8146250]

Treon M. Revised stuttering and related TPNB-LPPD threshold syndrome hypotheses in light of recent research. Psychology and Education: An Interdisciplinary Journal. 2010; 47(3 \& 4):1-27. Monograph.

Utendale WT, Nuselovici J, Saint-Pierre AB, Hubert M, Chochol C, Hastings PD. Associations between inhibitory control, respiratory sinus arrhythmia, and externalizing problems in early childhood. Developmental Psychobiology. 2014; 56(4):686-699. [PubMed: 23765984]

Walden TA, Frankel CB, Buhr AP, Johnson KN, Conture EG, Karrass JM. Dual Diathesis-Stressor Model of Emotional and Linguistic Contributions to Developmental Stuttering. Journal of Abnormal Child Psychology. 2012; 40:633-644. [PubMed: 22016200]

Wang FB, Holst M-C, Powley TL. The ratio of pre- to postganglionic neurons and related issues in the autonomic nervous system. Brain Research Reviews. 1995; 21(1):93-115. [PubMed: 8547955]

Weber CM, Smith A. Autonomic Correlates of Stuttering and Speech Assessed in a Range of Experimental Tasks. Journal of Speech and Hearing Research. 1990; 33:690-706. [PubMed: 2273884]

Weber EJ, Ven Der Molen MW, Molenaar PCM. Heart rate and sustained attention during childhood: Age changes in anticipatory heart rate, primary bradycardia, and respiratory sinus arrhythmia. Psychophysiology. 1994; 31:164-174. [PubMed: 8153252]

Wilder J. Basimetric approach (law of initial value) to biological rhythms. Annals of the New York Academy of Sciences. 1962; 98:1211-1220. [PubMed: 14000687]

Williams, K. Expressive Vocabulary Test (EVT). American Guidance Service, Inc; Circle Pines, MN: 1997.

Winkworth AL, Davis PJ. Breathing patterns during spontaneous speech. Journal of Speech \& Hearing Research. 1995; 38(1):124. [PubMed: 7731204] 
- We assessed autonomic activity of children who do (CWS) and do not stutter (CWNS).

- CWS exhibited lower respiratory sinus arrhythmia (RSA) than CWNS at baseline.

- CWS had greater skin conductance (SC) during speech following a positive condition.

- During speech CWS exhibited a significant positive relation between RSA and SC.

- CWS had greater increase of RSA and SC during emotion conditions than CWNS. 


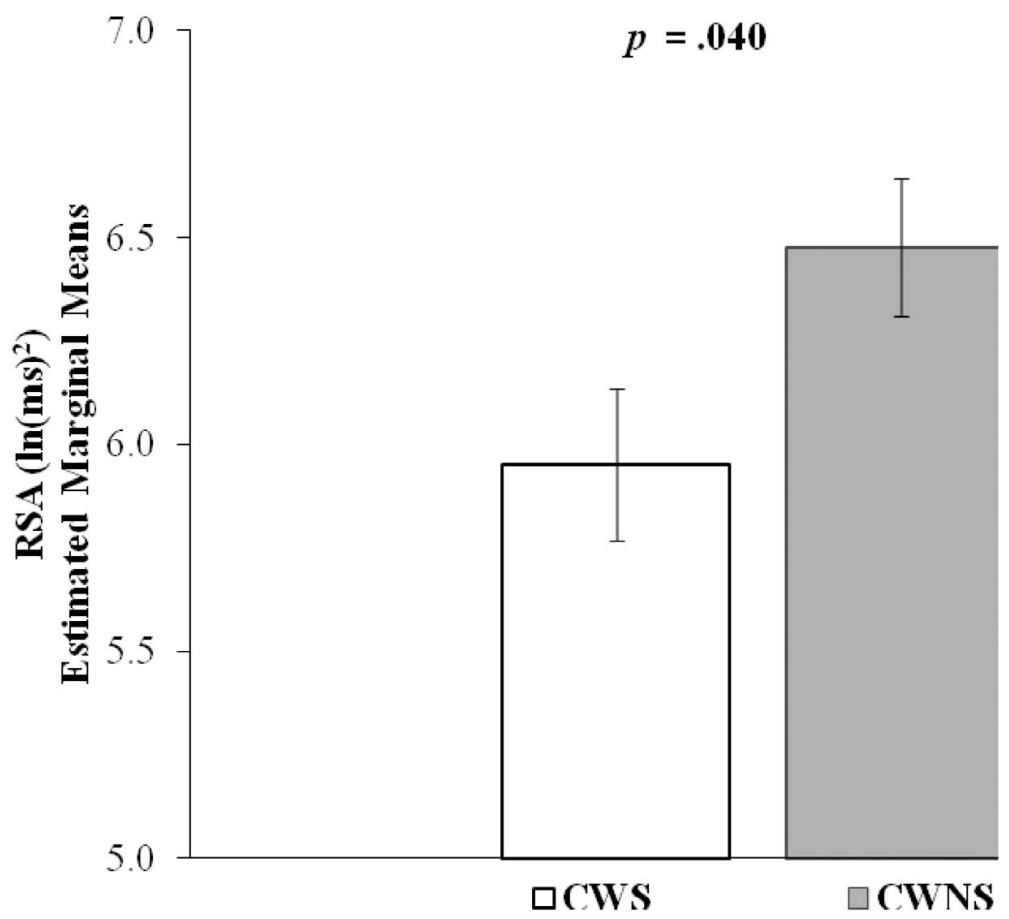

Figure 1. Respiratory sinus arrhythmia (RSA): Baseline Respiratory sinus arrhythmia (RSA) at baseline for preschool-age CWS ( $\mathrm{n}=20,15$ male) and CWNS ( $\mathrm{n}=21,11$ male) ( \pm standard error). 


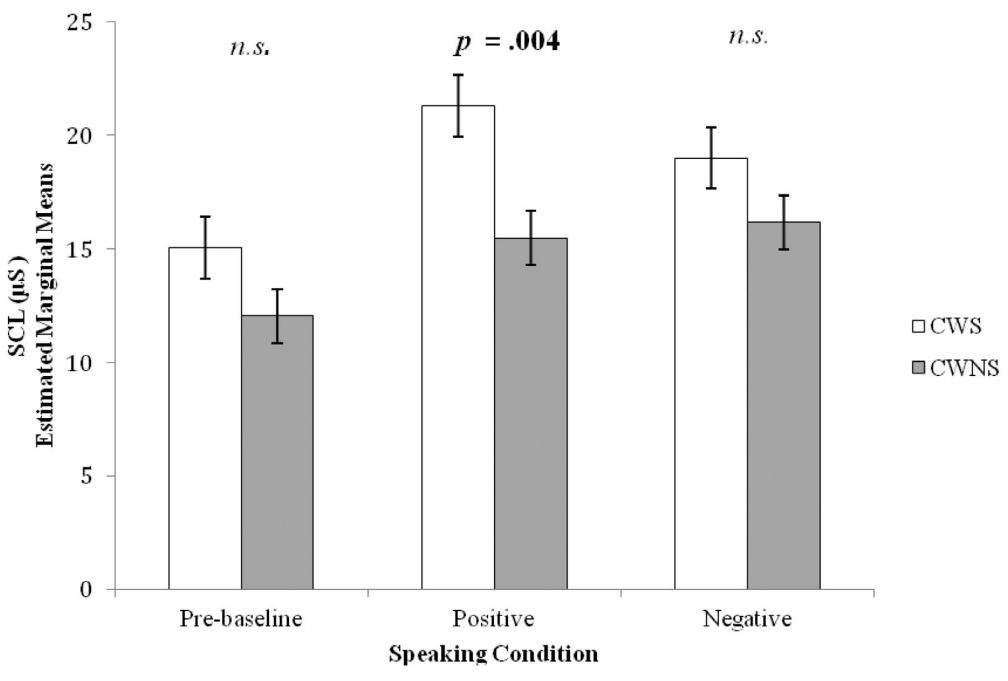

Figure 2. Skin Conductance Level (SCL): Speaking

Skin conductance level (SCL) during speaking for preschool-age CWS and CWNS ( \pm standard error). n.s. = non-significant 


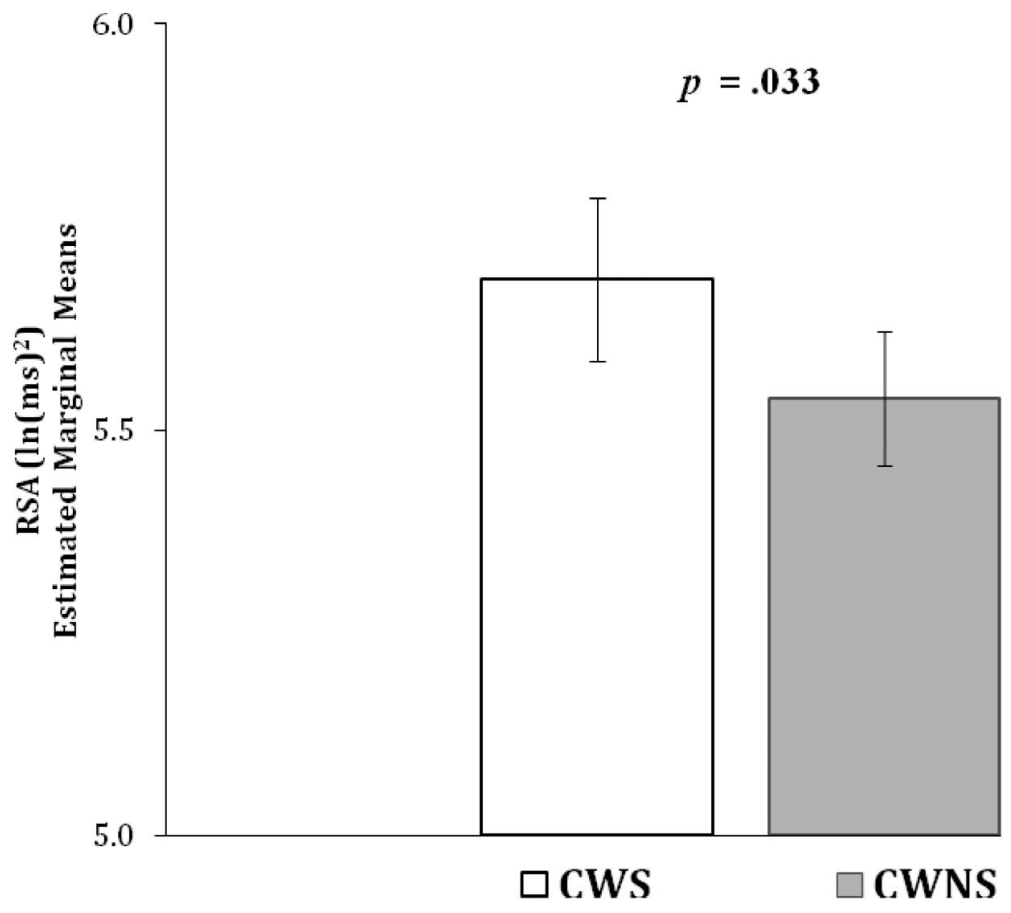

Figure 3. Respiratory sinus arrhythmia (RSA): Speaking

Respiratory sinus arrhythmia (RSA) during speaking conditions for preschool-age CWS and CWNS ( \pm standard error). 


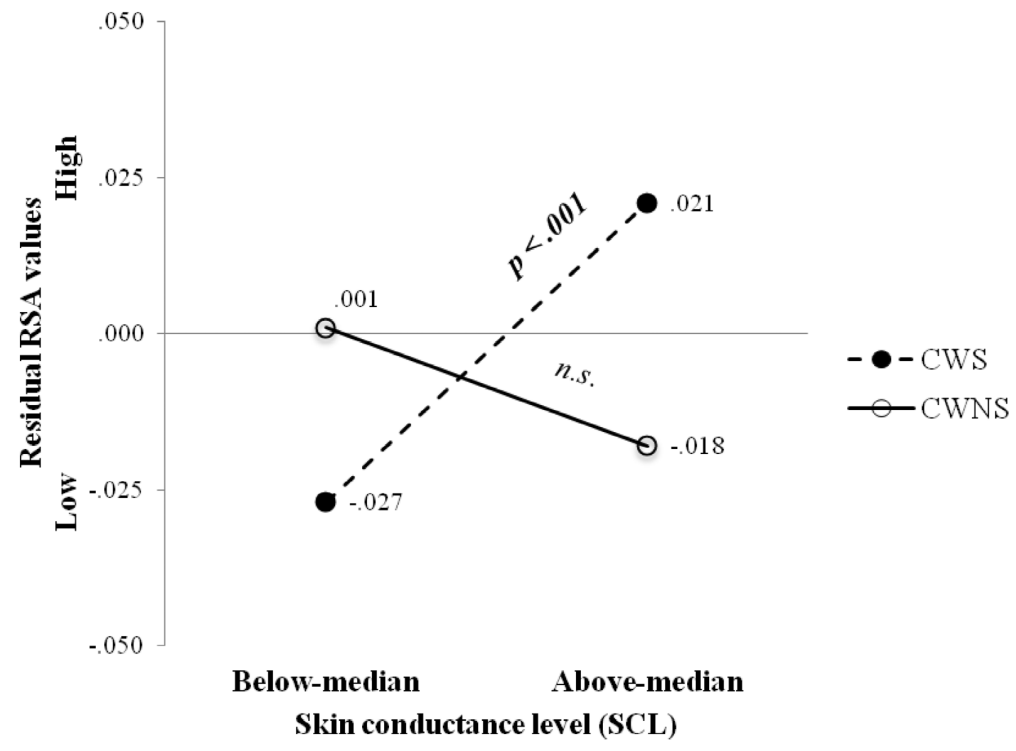

Figure 4. Relation of respiratory sinus arrhythmia (RSA) to skin conductance level (SCL): Speaking

Residual respiratory sinus arrhythmia (RSA) values from the speaking linear mixed-effects model for preschool-age CWS and CWNS with skin conductance level (SCL) below and above the median values. Values beside each closed and open circle = residual RSA values for each talker group. n.s. = non-significant

Note. Groups were dichotomized into SCL values below and above the median following inferential assessment in order to graphically depict the result. 


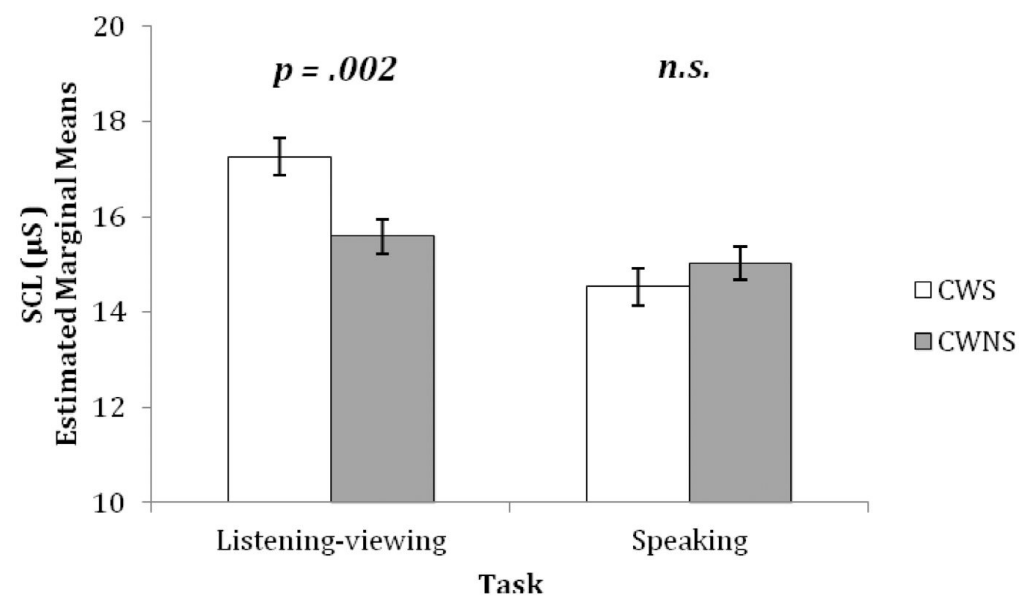

Figure 5. Skin conductance level (SCL): Listening-viewing versus speaking

Skin conductance level (SCL): between-group interaction effect for Listening-Viewing versus Speaking for preschool-age CWS and CWNS ( \pm standard error). n.s. = nonsignificant 


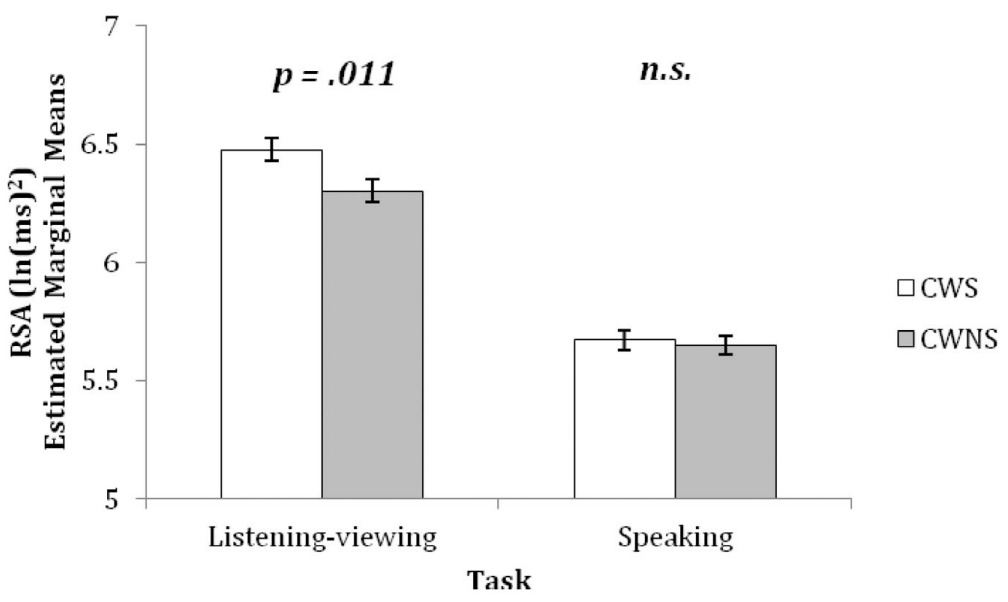

Figure 6. Respiratory sinus arrhythmia (RSA): Listening-viewing versus speaking Respiratory sinus arrhythmia (RSA): between-group interaction effect for ListeningViewing versus Speaking for preschool-age CWS and CWNS ( \pm standard error). n.s. = nonsignificant 




J Fluency Disord. Author manuscript; available in PMC 2015 September 01. 


\section{Table 2}

Descriptive statistics for respiratory sinus arrhythmia (RSA) and skin conductance level (SCL) for preschoolage children who stutter (CWS) and children who do not stutter (CWNS).

\begin{tabular}{|c|c|c|c|c|}
\hline \multirow[b]{2}{*}{ Conditions } & \multicolumn{2}{|c|}{ Respiratory Sinus Arrhythmia } & \multicolumn{2}{|c|}{ Skin Conductance Level } \\
\hline & $M(S D)$ & Range & $M(S D)$ & Range \\
\hline & \multicolumn{4}{|c|}{ Children who Stutter } \\
\hline Pre-baseline & $6.02(1.26)$ & $4.13-8.45$ & $10.71(7.13)$ & $1.91-29.02$ \\
\hline Post-baseline & $5.81(1.32)$ & $3.54-8.56$ & $19.78(10.49)$ & $3.08-38.69$ \\
\hline Negative & $6.06(1.41)$ & $3.85-9.01$ & $17.12(10.05)$ & $3.71-37.45$ \\
\hline Positive & $6.04(1.54)$ & $3.32-8.63$ & $18.22(10.24)$ & $4.36-38.47$ \\
\hline Narrative \#1 & $5.53(1.27)$ & $3.84-8.28$ & $13.18(8.12)$ & $1.64-31.73$ \\
\hline Narrative \#2 & $5.21(1.27)$ & $3.08-7.37$ & $17.13(9.61)$ & $5.33-37.67$ \\
\hline \multirow[t]{2}{*}{ Narrative \#3 } & $5.24(1.25)$ & $3.33-7.82$ & $19.52(10.62)$ & $3.93-37.51$ \\
\hline & \multicolumn{2}{|c|}{ Respiratory Sinus Arrhythmia } & \multicolumn{2}{|c|}{$\underline{\text { Skin Conductance Level }}$} \\
\hline \multirow[t]{2}{*}{ Conditions } & $M(S D)$ & Range & $M(S D)$ & Range \\
\hline & \multicolumn{4}{|c|}{ Children who do not Stutter } \\
\hline Pre-baseline & $6.72(.88)$ & $5.45-8.63$ & $9.90(5.51)$ & $1.87-22.59$ \\
\hline Post-baseline & $6.43(.96)$ & $4.78-7.93$ & $17.53(7.84)$ & $4.83-39.92$ \\
\hline Negative & $6.47(.90)$ & $5.20-8.30$ & $15.50(7.14)$ & $4.56-37.83$ \\
\hline Positive & $6.66(.84)$ & $5.44-8.18$ & $14.63(6.19)$ & $5.06-30.90$ \\
\hline Narrative \#1 & $6.16(.78)$ & $4.93-7.62$ & $12.59(5.27)$ & $3.06-25.09$ \\
\hline Narrative \#2 & $5.84(.70)$ & $4.88-7.18$ & $15.67(6.76)$ & $4.30-31.02$ \\
\hline Narrative \#3 & $5.97(.71)$ & $4.88-7.39$ & $17.37(7.02)$ & $5.90-37.32$ \\
\hline
\end{tabular}

Note: For the purposes of description, this table presents raw means and standard deviations. For the purposes of statistical analyses, however, estimated marginal means (EMM) and standard errors (SE) were used and reported in the prose description of findings. 
